\title{
REVIEW
}

Open Access

\section{A systematic review and meta-analysis of school-based interventions with health education to reduce body mass index in adolescents aged 10 to 19 years}

Chandni Maria Jacob ${ }^{1,2,3^{*+}}$ @ , Polly Louise Hardy-Johnson ${ }^{1,4^{*+}}$, Hazel M. Inskip ${ }^{2,4}$, Taylor Morris ${ }^{4}$, Camille M. Parsons ${ }^{4}$, Millie Barrett ${ }^{4}$, Mark Hanson 1,2,3, Kathryn Woods-Townsend $d^{2,5+}$ and Janis Baird ${ }^{2,4+}$

\begin{abstract}
Background: Adolescents are increasingly susceptible to obesity, and thus at risk of later non-communicable diseases, due to changes in food choices, physical activity levels and exposure to an obesogenic environment. This review aimed to synthesize the literature investigating the effectiveness of health education interventions delivered in school settings to prevent overweight and obesity and/ or reduce BMI in adolescents, and to explore the key features of effectiveness.

Methods: A systematic search of electronic databases including MEDLINE, CINAHL, PsychINFO and ERIC for papers published from Jan 2006 was carried out in 2020, following PRISMA guidelines. Studies that evaluated health education interventions in 10-19-year-olds delivered in schools in high-income countries, with a control group and reported BMI/BMI Z-score were selected. Three researchers screened titles and abstracts, conducted data extraction and assessed quality of the full text publications. A third of the papers from each set were cross-checked by another reviewer. A meta-analysis of a sub-set of studies was conducted for BMI z-score.

(Continued on next page)
\end{abstract}

\footnotetext{
*Correspondence: C.M.Jacob@soton.ac.uk; phj@mrc.soton.ac.uk

${ }^{\dagger}$ Chandni Maria Jacob and Polly Louise Hardy-Johnson are Joint first authors:

both first authors contributed equally to submission

${ }^{\dagger}$ Kathryn Woods-Townsend and Janis Baird are Joint senior authors

${ }^{1}$ Academic Unit of Human Development and Health, Faculty of Medicine, University of Southampton, Southampton, UK

Full list of author information is available at the end of the article
}

C C The Author(s). 2021 Open Access This article is licensed under a Creative Commons Attribution 4.0 International License, which permits use, sharing, adaptation, distribution and reproduction in any medium or format, as long as you give appropriate credit to the original author(s) and the source, provide a link to the Creative Commons licence, and indicate if changes were made. The images or other third party material in this article are included in the article's Creative Commons licence, unless indicated otherwise in a credit line to the material. If material is not included in the article's Creative Commons licence and your intended use is not permitted by statutory regulation or exceeds the permitted use, you will need to obtain permission directly from the copyright holder. To view a copy of this licence, visit http://creativecommons.org/licenses/by/4.0/ The Creative Commons Public Domain Dedication waiver (http://creativecommons.org/publicdomain/zero/1.0/) applies to the data made available in this article, unless otherwise stated in a credit line to the data. 
(Continued from previous page)

Results: Thirty-three interventions based on 39 publications were included in the review. Most studies evaluated multi-component interventions using health education to improve behaviours related to diet, physical activity and body composition measures. Fourteen interventions were associated with reduced BMI/BMI z-score. Most interventions $(n=22)$ were delivered by teachers in classroom settings, 19 of which trained teachers before the intervention. The multi-component interventions $(n=26)$ included strategies such as environment modifications $(n=10)$, digital interventions $(n=15)$ and parent involvement $(n=16)$. Fourteen studies had a low risk of bias, followed by 10 with medium and nine with a high risk of bias. Fourteen studies were included in a random-effects meta-analysis for BMI z-score. The pooled estimate of this meta-analysis showed a small difference between intervention and control in change in BMI z-score $(-0.06$ [95\% Cl -0.10, - 0.03]). A funnel plot indicated that some degree of publication bias was operating, and hence the effect size might be inflated.

Conclusions: Findings from our review suggest that school-based health education interventions have the public health potential to lower BMI towards a healthier range in adolescents. Multi-component interventions involving key stakeholders such as teachers and parents and digital components are a promising strategy.

Keywords: Adolescent health, Body mass index, Obesity, School, Health education, Physical activity, Diet, Nutrition, Intervention

\section{Background}

Approximately 340 million children and adolescents aged 5-19 years had overweight or obesity globally in 2016 [1]. Almost 80\% of adolescents with obesity will have obesity as adults [2] and the prevalence of morbid obesity in adults is higher among those who had obesity as adolescents [3]. Obesity in childhood and adolescence is associated with an increased risk of noncommunicable diseases (NCDs) such as Type 2 diabetes, cardiovascular disease, chronic obstructive lung disease and some forms of cancer [4]. Adolescents with excess weight or obesity often have decreased self-esteem and may be subjected to bullying and discrimination, increasing the risk of poor psychological health and eating disorders $[5,6]$.

Adolescence is a transitional period characterized by critical changes in body composition, insulin sensitivity, health behaviours and psychological and social functioning, as well as increased autonomy [5, 7]. The likelihood of unhealthy eating behaviours, high screen-time, disordered sleep patterns and decreased participation in physical activity (especially among girls) increases during adolescence [8-11]. Factors leading to adolescent obesity can be broadly categorized into individual (food preferences, taste and perceptions, self-efficacy for making healthy choices, and convenience), social (including family and peer relationships), demographic (socioeconomic status (SES)) and environmental (mass media, easy access to fast-food outlets and vending machines, lack of safe active recreation and travel options) factors [12, 13]. It has been suggested that obesity prevention interventions may be more effective in adolescents than younger children, as they are more likely to understand the concepts and have more autonomy, for example about food choices [14].
Previous systematic reviews covering the period before 2006 have evaluated the effectiveness of interventions to improve obesity-related outcomes such as body mass index (BMI), physical activity and dietary behaviours in children and adolescents together, or for particular countries [15-18]. However growing evidence suggests that the school setting provides a platform for effective and sustained intervention delivery to prevent overweight and obesity $[19,20]$. Such interventions have often incorporated methods such as health education, providing healthy school meals, parental involvement, and community engagement [15, 21-23]. As adolescents are already engaging in formal education and activities with their peers, schools provide an ideal platform for delivering health interventions, but most reviews examining the effects of school-based programmes on BMI have considered children and adolescents together. A meta-analysis $(n=5)$ showed no significant change in the BMI of children and adolescents receiving the intervention compared with control groups but data disaggregated for 2-19year-olds were not presented [16]. Fewer trials based in schools were specifically for adolescents, [22] but changing health behaviour patterns may mean that strategies to prevent obesity in this age group require a different approach from those used in younger children.

In the UK, increasing health education in schools has been recommended as part of the government's Childhood Obesity Strategy [24]. Health education, provided in daytime and after-school programmes, has been widely recommended as a tool to address obesity [25], by encouraging behaviour change and improved health literacy [26]. However, to date, reported effects of health education on body composition and weight have been mixed, possibly due to the short duration of 
interventions and a lack of attention to accompanying lifestyle changes outside the school environment [27]. The effectiveness of health education as a way of reducing BMI in adolescence has not been reviewed.

\section{Aim}

The aim of this review was to synthesize evidence to answer the following questions: 1) What is the effectiveness of health education interventions delivered in school settings to prevent overweight and obesity and/ or reduce BMI in adolescents? 2) What are the key features of effective interventions?

\section{Methods}

\section{Search strategy}

A comprehensive and systematic search of published literature was undertaken in January 2017, and updated in June 2020, in accordance with the Preferred Reporting Items for Systematic reviews and Meta-Analysis (PRIS MA) [28] and the University of York Centre for Reviews and Dissemination (CRD) Guidelines [29]. We focused our searches on the period since 2006 which was not covered by existing high-quality systematic reviews of school-based interventions. The following electronic databases were searched in full: MEDLINE, ERIC, PsychINFO and CINAHL. A combination of medical subject headings (MESH) and free text keywords were used to find intervention studies, limited to English language and published between January 2006 and 2020. Separate search strings were developed for diet (e.g., fruit), physical activity (e.g., exercise, sport) and obesity (e.g., overweight, obese), intervention (e.g., education, health literacy) and adolescents (e.g., teen, youth) (see Supplementary Material 1). We consulted an information specialist (LP) to review and comment on the search strategy and contacted experts in the field to identify studies not located in the database searches. Reference lists of included articles were also screened. The proposal for this review was registered with PROSPERO (ID: CRD42016053477).

\section{Selection criteria}

Experimental studies that investigated the effectiveness of health education interventions in adolescents aged 10-19 years in school settings [30], and which reported BMI and/or BMI z-scores as outcomes [31] were included in this review. Table 1 presents further details on the rationale for the inclusion criteria. Included studies needed to have a control or comparison group and pre/ post-intervention measures for BMI outcomes (at least one post-intervention measure). BMI and BMI z-score were selected as they are commonly used for assessing overweight and obesity in adolescents. Differences in education systems, modes of delivery of interventions, cultural and contextual differences could affect the relevance and applicability of the findings. Therefore, only studies from high-income countries were included in this review. The definitions for income groups for countries by World Bank (2020) [32] were used to exclude low-and middle-income countries (LMICs). We also included multi-component intervention studies that addressed other issues such as unhealthy diet and physical inactivity if they also reported BMI outcomes. Health education was defined as 'any combination of learning experiences designed to facilitate voluntary adaptations of behaviour conducive to health' [33]. By this definition, interventions delivered in an educational setting, which provided information on improving diet and/or physical activity and preventing excess weight gain were included. Educational interventions supplemented by behaviour change techniques and using innovative tools for dissemination such as digital interventions were also included. As schools often have general health education as part of their curriculum, only interventions that were delivered as an addition to existing lessons, with the main component delivered within the school environment, were included. Studies focusing only on specific groups (e.g. adolescents with obesity, or specific medical conditions) were excluded.

\section{Data extraction and quality assessment}

Titles and abstracts were downloaded, and duplicates were removed using EndNote bibliographic software. Three authors (CMJ, PHJ and MB) screened titles and abstracts that met the inclusion criteria. Full texts were then assessed for eligibility by three reviewers (CMJ, $\mathrm{PHJ}, \mathrm{MB}$ ) and any disagreements resolved through discussion with a fourth reviewer (JB). Data were extracted from included studies using a form to capture key information on populations, intervention strategies and results. A modified version of a quality assessment rubric, based on CRD guidance, was used to assess risk of bias in included studies in relation to the review questions. Risk of bias scores ranged from -6 to +11 and were categorized into low risk ( +5 and above), medium risk $(+1$ to +4$)$ and high risk $(0$ to -6$)$. Scores of $+1 / 0 /-1$ were given for different criteria (e.g. selection criteria, analytical methods) and tallied to provide a final score for risk of bias. For example, studies were awarded +1 for randomized controlled trials, 0 for quasi-experimental studies that include a control group, and -1 for experimental studies that do not use a control group. A full description of the assessment criteria can be found in the supplementary material. The included studies were divided into two sets, and reviewers (CMJ and $\mathrm{PHJ}$ ) reviewed one set each. Papers identified through an updated search (2018-2020) were reviewed by three reviewers (PHJ, CMJ and $\mathrm{MB}$ ). To ensure consistency, a 
Table 1 Review inclusion and exclusion criteria

\begin{tabular}{|c|c|c|}
\hline & Criteria & Justification \\
\hline \multirow[t]{5}{*}{ Inclusion } & $\begin{array}{l}\text { Observational and experimental studies with a control or } \\
\text { comparison group }\end{array}$ & $\begin{array}{l}\text { We aimed to provide a thorough systematic review of recent literature. } \\
\text { Though the quality of evidence will be lower for observational studies a } \\
\text { detailed and transparent quality assessment of included studies was } \\
\text { conducted. }\end{array}$ \\
\hline & Participants within the specified age range of 10-19. & The review focuses on adolescents only. \\
\hline & $\begin{array}{l}\text { Studies that report BMI and related outcomes with a pre } \\
\text { and post-intervention comparison, baseline to follow up etc. }\end{array}$ & To identify interventions that bring about a change in outcomes \\
\hline & High-income countries & $\begin{array}{l}\text { Differences in education systems, modes of delivery of interventions, } \\
\text { cultural and contextual differences, co-existence of under-and over-nutrition } \\
\text { could all affect the relevance and generalisability of the findings. The defini- } \\
\text { tions for income groups for countries by World bank (2020) were used to } \\
\text { exclude low and middle-income countries. }\end{array}$ \\
\hline & Interventions from year 2006 & $\begin{array}{l}\text { High quality systematic reviews covered the evidence of school-based inter- } \\
\text { ventions up to } 2006 \text {. We aimed to provide an updated account of interven- } \\
\text { tions with focus on adolescents in school settings. }\end{array}$ \\
\hline \multirow[t]{3}{*}{ Exclusion } & Studies that do not report change in BMI outcomes & $\begin{array}{l}\text { Studies that aim to prevent obesity and overweight or reduce BMI were } \\
\text { included. BMI outcomes (BMI, BMI Z-score, change in prevalence of over- } \\
\text { weight and obesity) were selected as the outcomes for this review as an ini- } \\
\text { tial scoping search showed that BMI/ BMI Z score were most commonly } \\
\text { reported in studies based in schools. BMI is also more feasible for assess- } \\
\text { ment of students in schools. }\end{array}$ \\
\hline & Papers published in a language other than English & Translation resources not available \\
\hline & $\begin{array}{l}\text { Specific disease groups targeted (e.g. Diabetes and other } \\
\text { endocrine disorders) } \\
\text { Interventions targeting only students with overweight and/ } \\
\text { or obesity at baseline }\end{array}$ & $\begin{array}{l}\text { The review aims to explore interventions with implications for the general } \\
\text { population and targeted interventions for different conditions may require } \\
\text { different strategies. }\end{array}$ \\
\hline
\end{tabular}

third of the papers from each set were cross-checked by another reviewer.

\section{Statistical analysis}

A meta-analysis was conducted for studies presenting data on BMI z-score. Although some issues were present due to heterogeneity of target groups, specific intervention components and how outcome measures were presented, sufficient data were available for meta-analysis in 14 of the 33 included studies [34-47]. Studies not included in the meta-analysis did not provide results for BMI z-score but reported a change in BMI, BMI percentile or prevalence of obesity/ overweight based on calculated BMI or BMI z-score. Heterogeneity was assessed using Cochran's $Q$ and the percentage of variability due to heterogeneity was quantified using $\mathrm{I}^{2}$. To account for the heterogeneity between studies, a random-effects model was used in the meta-analysis. Where follow-up results were recorded at different time points during data extraction, the longest follow-up measure was used, and, where available, sub-group results (based on gender) were obtained. Meta-analysis was conducted on the full dataset from the 14 studies and then repeated according to gender for those studies that reported such findings separately. Due to the limited number of studies eligible for meta-analysis, subset analyses by intervention features, risk of bias and mode of delivery could not be conducted. Funnel plots were created to assess the possibility of publication bias for studies included in the meta-analysis $(n=14)$. All analyses were performed using Stata version 14 (StataCorp LP, College Station, Texas, USA).

\section{Results}

\section{Results of literature search}

The total search retrieved 34,772 records. Following removal of duplicates, 32,828 were screened by title and abstract. The remaining 363 full text articles were screened, of which 39 publications met the inclusion criteria (see Fig. 1 PRISMA flow chart). Some of the publications were based on the same study cohorts but reported different outcomes of the same intervention. Where this occurred, we grouped the publications by study cohort for reporting and analysis.

\section{Characteristics of included studies}

We identified 39 papers, based on 33 studies [34-67]. Six studies recruited adolescent girls only [36, 40, 44, 47, $61,63]$ one adolescent boys only [42] and one study included parent-student dyads [66]. Most of the studies $(n=27)$ focused on adolescents aged 10-14 years [34, $36-42,44-48,50,52,54,56-66]$, and six recruited participants from high schools/secondary schools without defining an age range [35, 43, 49, 51, 53, 55]. Eighteen 


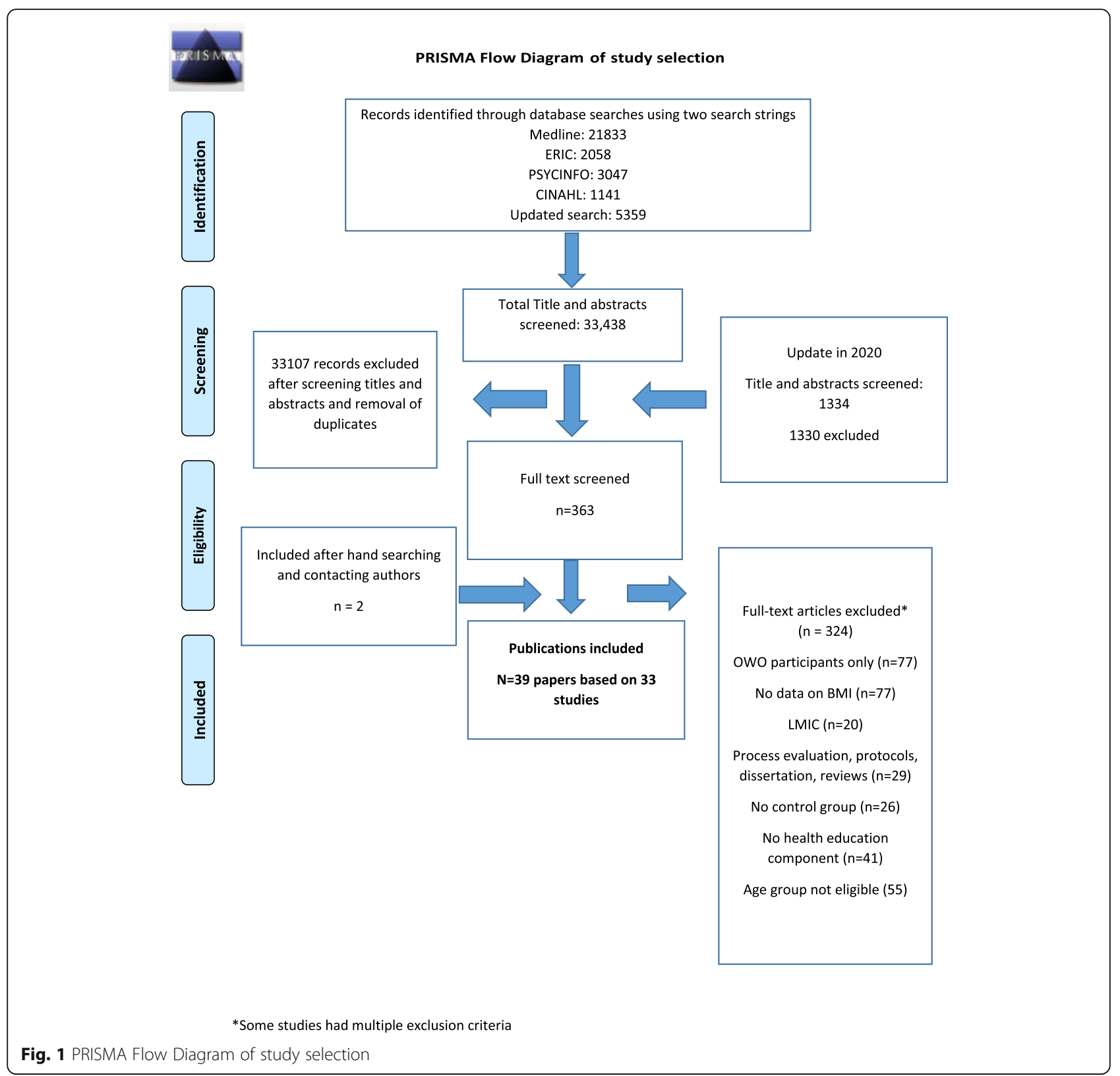

reported the use of behaviour change theory to inform the development of their interventions [36, 38, 40-42, 44, 47, $52-57,61,63-66]$ with the most common theory being social cognitive theory $[36,38,41,42,52,53,55,56,61]$.

Most studies evaluated multi-component interventions which, in addition to health education delivered in the classroom, included components such as homework activities, environment modification, physical activity classes, and fruit and vegetable breaks during class $(n=$ 26). The remaining seven interventions included health education interventions only [34, 45, 50, 60, 62, 64, 65]. Table 2 provides an overview of the features of included studies and percentage of effectiveness for each component.
Thirteen of the studies included had an after-school component such as monitoring diet and physical activity habits at home, providing additional resources to support behaviour change at home (e.g., recipe cards), community activities and social events $[35,36,38,40,43$, $46-48,55,57,59,63,65,66]$. Twelve studies provided additional sessions for organised sports or clubs to increase exercise or physical activity [36, 42-44, 47, 5153, 56, 58, 63, 64]. Table 3 describes the characteristics of each intervention. Quality assessment scores are reported in Table 4. A quality assessment of the 33 studies included in this review indicated that 14 had a low risk of bias compared with 10 with medium and nine with a high risk of bias. Risk of Bias scores ranged from -3 [49, 
Table 2 Key characteristics of studies included in the review

\begin{tabular}{|c|c|c|c|}
\hline Characteristic & Citations & $\begin{array}{l}\text { Proportion of Studies N (\% } \\
\text { of } 33 \text { ) }\end{array}$ & $\begin{array}{l}\mathrm{N} \text { and \% with } \\
\text { Significant BMI Outcomes }\end{array}$ \\
\hline \multicolumn{4}{|l|}{ Intervention delivery } \\
\hline Teachers & {$[34-39,45,46,48-50,52-54,57,59,61,62]$} & $22(67 \%)$ & $12(54 \%)$ \\
\hline Researchers & {$[55,56,58,63-65]$} & $6(18 \%)$ & $2(33 \%)$ \\
\hline School Nurse & [44] & $1(3 \%)$ & 0 \\
\hline School Project Officer & [43] & $1(3 \%)$ & $1(100 \%)$ \\
\hline Community based instructor & {$[47,66]$} & $2(6 \%)$ & 0 \\
\hline Not Stated & [51] & $1(3 \%)$ & 0 \\
\hline CPD for teachers Provided & {$[34,36-43,45,48-50,52-54,57,59,61]$} & $19(58 \%)$ & $12(63 \%)$ \\
\hline \multicolumn{4}{|l|}{ Mode of CPD ${ }^{\mathrm{a}}$} \\
\hline Textbook/Manual & {$[37,39,50,52,54,57,59]$} & $7(21 \%)$ & $3(43 \%)$ \\
\hline Seminars/Workshop & {$[36,38,40-43,53,54,61]$} & $9(27 \%)$ & $6(67 \%)$ \\
\hline Training on Intervention Delivery only & {$[34,38,39,45,52]$} & $5(15 \%)$ & $3(60 \%)$ \\
\hline Not stated & {$[48,49]$} & $2(6 \%)$ & $1(50 \%)$ \\
\hline Included Parental Involvement & $\begin{array}{l}{[36,37,39,40,42,43,46,48,49,52-56,63} \\
66]\end{array}$ & $16(48 \%)$ & $9(56 \%)$ \\
\hline \multicolumn{4}{|l|}{ Mode of Parental Involvement } \\
\hline Text messages/ Emails & {$[37,46]$} & $2(6 \%)$ & $1(50 \%)$ \\
\hline Newsletters/Information leaflets & {$[36,40,42,43,49,52,53,56]$} & $8(24 \%)$ & $5(63 \%)$ \\
\hline Family workshops/ Parent Engagement & {$[39,48,54,55,63,66]$} & $6(18 \%)$ & $2(33 \%)$ \\
\hline \multicolumn{4}{|l|}{ Engagement components $^{\mathrm{b}}$} \\
\hline $\begin{array}{l}\text { App/Website/CD-ROM/ text messages/ } \\
\text { social media }\end{array}$ & {$[35,36,40,42,43,46,50,55,59,63-66]$} & $15(45 \%)$ & $5(83 \%)$ \\
\hline Computer-Tailored Advice & {$[40,47,50,57,59]$} & $5(15 \%)$ & $1(20 \%)$ \\
\hline After-school/out of class & {$[35,36,38,40-44,46,48,55,59,63]$} & $13(39 \%)$ & $5(38 \%)$ \\
\hline Games & {$[34,45]$} & $2(6 \%)$ & $1(50 \%)$ \\
\hline \multicolumn{4}{|l|}{ Other Intervention Features } \\
\hline $\begin{array}{l}\text { Environmental modification/ School } \\
\text { Policy Change }\end{array}$ & {$[37,39,40,42,46,48,49,52,59,61]$} & $10(30 \%)$ & $6(60 \%)$ \\
\hline Community Programme & {$[43,55]$} & $2(6 \%)$ & $1(50 \%)$ \\
\hline Exercise/ PA classes & {$[36,42-44,47,51-53,56,58,63,66]$} & $12(36 \%)$ & $4(33 \%)$ \\
\hline \multicolumn{4}{|l|}{ Study Design } \\
\hline $\mathrm{RCT}$ & {$[34-36,38-42,45-48,50,52-59,62,63]$} & $23(70 \%)$ & $9(39 \%)$ \\
\hline Non-RCT & {$[37,41,43,49,51,60,61,64-66]$} & $10(30 \%)$ & $5(50 \%)$ \\
\hline \multicolumn{4}{|l|}{ Intervention Duration } \\
\hline$<6$ months & $\begin{array}{l}{[34,38,41,42,45,47,48,50,53-56,58,60,} \\
62,65,66]\end{array}$ & $17(52 \%)$ & $8(47 \%)$ \\
\hline 6 months- 1 year & {$[36,44,51,59,63]$} & $5(15 \%)$ & 0 \\
\hline $1-2$ years & {$[35,37,39,40,49,57,61,64]$} & $8(24 \%)$ & $4(50 \%)$ \\
\hline$>2$ years & {$[43,46,52]$} & $3(9 \%)$ & $2(67 \%)$ \\
\hline \multicolumn{4}{|l|}{ Intervention type } \\
\hline Health education only & {$[34,45,50,60,62,64,65]$} & $7(21 \%)$ & $2(29 \%)$ \\
\hline Multi-component & {$[35-45,46-49,51-59,61,63,66]$} & $26(79 \%)[67]$ & $12(48 \%)$ \\
\hline
\end{tabular}

${ }^{a}$ Training on intervention delivery was provided for all interventions. Additional training was also provided for some studies, indicated in the table ${ }^{\mathrm{b}}$ Some studies may include more than one facilitator, delivery mode or intervention strategy 
Table 3 Summary of findings from included studies

\begin{tabular}{|c|c|c|c|}
\hline $\begin{array}{l}\text { Author, Year, Country, } \\
\text { Name of Cohort/ study, } \\
\text { Citation }\end{array}$ & $\begin{array}{l}\text { Study design, Participant } \\
\text { characteristics }^{\mathrm{a}}\end{array}$ & Key Intervention characteristics & $\begin{array}{l}\text { Main findings and results for BMI/BMI z- } \\
\text { score outcomes }{ }^{b}\end{array}$ \\
\hline
\end{tabular}

\section{Low Risk of Bias}

Robbins, 2020 [66], USA GOAL

Robbins 2020 [67], USA and Pfeiffer et al. (2019) [47], USA Girls on the Move

Wadolawska, 2019 [60] Poland

Bogart, $2016^{\mathrm{d}}[48]$ USA

Lubans, 2016 [42] and Smith 2014 [68],

Australia

ATLAS

Melnyk, 2015 [53], USA COPE

Viggiano, 2015 [45], Italy ${ }^{c} \quad$ RCT
Pre test-post-test quasiexperimental design Mean age $11.6(0.09)$ Intervention: $n=38$ Control: $n=43$ A total of 81 parentstudent dyads were recruited

Group RCT

Mean age 12.07 (girls)

Intervention: $n=593$

Control: $n=601$ (schools in low-income areas)
- Primary outcomes: feasibility and acceptability (qualitative), MVPA, diet quality, psychosocial variables (motivation, self-efficacy, and perceived social support for PA and healthy eating

- Duration of intervention: 12 weeks

- Follow up post intervention

- Teacher training: not needed as delivered by instructors and managers with experience in nutrition programmes (for the after-school clubs)

- Parental involvement: parent- student dyad combined meetings

- Digital component: Facebook participation with weekly healthy eating and PA habit forming tasks for parents to help adolescents with MVPA and diet

- Behaviour change theory: Self-determination theory and information-motivation-behaviour skills (IMB) model

- Primary outcome: MVPA, BMI Z score, Percentage body fat and cardiorespiratory fitness

- Duration of intervention: 17 weeks

- Follow up: immediate post-intervention and 1-4 weeks

after intervention was completed

- Delivered by trained nurse and community-based

instructors

- Digital component: one interactive internet-based session providing motivational and feedback messages

- Parental involvement: None during intervention period

- Behaviour Change Theory: Health promotion model and Self-determination theory
Non-randomised study with control group Mean age: $11.9(11.9-12.0)$ Intervention $n=319$ Control $n=145$

\section{RCT}

Mean age: $12.2(0.68)$

Intervention: $n=829$

Control: $n=539$

Cluster RCT

Mean age: $12.7(0.5)$

Intervention: $n=121$

Control: $n=143$
Cluster RCT

Mean age: $14.74(0.73)$ Intervention: $n=358$

Control: $n=42$
- Primary outcome: Physical activity, sedentary time, diet and body composition (z-Waist to height Ratio, Z BMl for age, Waist circumference)

- Duration of intervention: 3 weeks

- Follow up: 9 months after baseline

- Delivered by researchers

- Digital component: None

- Parental involvement: None

- Behaviour change theory: Integrated theory

- Primary outcome: BMI percentile

- Duration of intervention: 5 weeks

- Follow up after intervention: 2 years

- Teacher training: Delivered by peer leaders and teachers (training given to peer leaders).

- Parental involvement: Homework activities with parents

- Behaviour change theory: Social cognitive theory and community based participatory research

- Primary outcome: BMI, waist circumference

- Duration of intervention: 8 months

- Follow up: immediately after intervention and 18 months

from baseline

- Teacher training: two 6-h workshops

- Digital component: smartphone app and website.

- Parental involvement: Parent newsletters.

- Behaviour change theory: self-determination theory and social cognitive theory

- Primary outcome: BMI and depressive symptoms

- Intervention duration: 15 weeks

- Follow up after intervention: 12 months

- Teacher training: full day training workshop

- Digital component: None reported

- Parental involvement: Newsletter provided and students

were expected to discuss with parents

- Behaviour change theory: cognitive theory

- Primary outcomes: dietary behaviours and BMI z-scores
Proportion of overweight and obese: Intervention group: Baseline and post intervention 55.9\% ( $p=1.00)$

Control group

Baseline 40.5\%

Follow up $50.0 \%(p=0.13)$

No significant differences occurred for BMI z score post intervention $(B=-0.02, P=0.232$ ) Intervention

Baseline 1.30 (0.74)

Post intervention $1.30(0.74)$

Control

Baseline $1.42(0.73)$

Follow up $1.44(0.73)$

Unadjusted effect Size Cohen $\mathrm{d}=-0.03$

(subset analysis based on attendance (days/

week) provided but did not show any effect on BMl outcomes.

1-4 week Follow up results:

Control (766): 12.05 years.

Intervention (753): 12.05 years.

No significant between-group differences in

BMI-z existed at post intervention $(B=-0.02$,

.191, 95\% confidence

interval $[\mathrm{Cl} ;-0.05-0.01])$

Change in z-BMI-for-age SDs (Follow up

baseline):

Int: $-0.01(-0.07$ to 0.04$)$

Con: $0.03(-0.01$ to 0.07$)$

Difference: -0.04

$\mathrm{BMI}$ percentile (intervention versus control): adjusted difference $=-0.98$ (SE 1.01), $p=0.33$ Subset analysis (students obese at baseline) $\mathrm{BMI}$ percentile adjusted $\mathrm{b}=-2.33$ (SE 0.83; $P=$ 0.005) compared with control students.

BMI z-score (adjusted mean difference) at 18 months from baseline: 0.04 (95\%Cl: -0.07 to 0.14 )

COPE teens (24.94, SE 0.12)

Control group (25.48, SE 0.11)

$p$-value for difference $=0.001$ 
Table 3 Summary of findings from included studies (Continued)

\begin{tabular}{lll}
\hline $\begin{array}{l}\text { Author, Year, Country, } \\
\text { Name of Cohort/ study, } \\
\text { Citation }\end{array}$ & $\begin{array}{l}\text { Study design, Participant } \\
\text { characteristics }^{\mathbf{a}}\end{array}$ & Key Intervention characteristics \\
\hline Kaledo & Mean age $13.0(12.9-13.0)$ & - Duration of intervention: 20 weeks \\
& Intervention: $n=1663$ & - Follow up: 6 and 18 months post baseline \\
& Control: $n=1447$ & - Teacher training: teachers trained in playing the game \\
& - Digital component: None reported \\
& - Parental involvement: None reported \\
& - Behaviour change theory: Not specified
\end{tabular}

\section{Dewar, 2013 [36], \\ Australia}

Neat Girls

Ezendam, 2012 [50],

Netherlands

FATaintPHAT

Robbins, 2012 [44], USA
Pilot study Girls on the

Move

Prins, 2012 [57], Netherlands

The HEALTHY study group, 2010 [52], USA

Peralta, $2009[56]$ Australia FILA study (Fitness Improvement Lifestyle Awareness)

Singh, 2007 [59], The Netherlands DOiT (Dutch Obesity
RCT

Mean age $13.2(0.5)$ Intervention $n=178$ Control $n=179$

Girls only

RCT

Mean age (control group):

$12.7(0.7$

Control: 340

Intervention: 395

Quasi-experimental study Mean age: 11.4 years Intervention: $n=37$ Control: $n=32$
$\mathrm{RCT}$

Mean age: $12.7(0.5)$ Intervention: 281 Control: 254

RCT

Mean age $11.3(0.6)$ Intervention: 4603 Control: 2296

RCT

Mean age $12.5(0.4)$ Intervention: $n=16$ Control: $n=17$ Boys only

RCT

Mean age (control) 12.8 (0.51)
- Primary outcomes: BMI

- Duration of intervention: one year

- Follow up at 12 months and 24 months (12 months post intervention)

- Teacher training: 1-day training workshop.

- Digital component: SMS

- Parental involvement: Parent newsletters

- Behaviour change theory: Social cognitive theory

- Primary outcomes: Waist circumference, BMI and fitness

- Duration of intervention: 10 weeks

- Follow up at 4 months and 2 years

- Teacher training: manual provided

- Digital component: primarily an internet-based

intervention

- Parental involvement: None reported

- Behaviour change theory: Theory of Planned Behaviour

- Primary outcomes: BMI, physical activity, and cardiovascular fitness

- Duration of intervention: 6 months

- Follow up after intervention: immediately post

intervention

- Teacher training: Motivational Interviewing training for the school nurse

- Digital component: None reported

- Parental involvement: None reported

- Behaviour change theory: health promotion model

- Primary outcomes: Compliance with MVPA guideline and minutes spent in MVPA

- Duration of the intervention: Not reported

- Follow up at one and six months post intervention

- Teacher training: Manual provided

- Digital component: Computer tailored PA promotion

- Parental involvement: None reported

- Behaviour change theory: Socio-ecological model

- Primary outcomes: Risk of diabetes (BMl, waist circumference, fasting glucose and insulin levels), combined prevalence of OWO

- Duration of intervention: 3 years

- Follow up at 3 years from baseline

- Teacher training: $4 \mathrm{~h}$ training with companion manual

- Digital component: None

- Parental involvement: newsletters matching theme of semester

- Behaviour change theory: Social cognitive theory

- Primary outcome: BMI

- Follow up after intervention: 6 months

- Teacher training: None (delivered by researcher)

- Digital component: None reported

- Parental involvement: Newsletters

- Behaviour change theory: social cognitive theory

- Primary outcomes: BMI, measures of body fatness and aerobic fitness

- Duration of intervention: 8 months
Main findings and results for BMI/BMI zscore outcomes ${ }^{b}$

At 6 month follow-up:

Middle schools:

Intervention: 0.49 (95\%Cl: 0.45 to 0.53 )

Control: 0.58 (95\%Cl: 0.54 to 0.62$)$

$\mathrm{p}$-value for difference $=0.007$

High schools:

Intervention: 0.35 (95\%Cl: 0.29 to 0.40$)$

Control: 0.57 (95\%Cl: 0.51 to 0.63 )

$\mathrm{p}$-value for difference $<0.001$

At 18 month FU:

Middle schools:

Intervention: 0.40 (95\%Cl: 0.28 to 0.52$)$

Control: 0.57 ( $95 \% \mathrm{Cl}: 0.44$ to 0.71$)$

$\mathrm{p}$-value for difference $=0.057$

High schools:

Intervention: 0.13 ( $95 \% \mathrm{Cl}$ : -0.09 to 0.34$)$

Control: 0.61 ( $95 \% \mathrm{Cl} 0.31$ to 0.90$)$

$\mathrm{p}$-value for difference $=0.015$

Adjusted BMI z-score change at 12 month post intervention:

-0.12 (95\%Cl: $-0.27,0.04)$

Not reported for 4-month follow-up.

BMI change at 2 years between group difference: -0.14 (95\%Cl: -0.17 to 0.45 )

BMI z-score change

Intervention: $0.06(0.18)$

Control: 0.12 (0.18)

Adjusted difference: $-0.04(p=0.24)$

Unstandardized regression coefficient (95\% CI) for prevalence of overweight and obesity at 6 month follow-up: 0.16 (95\%Cl: -1.01 to 1.13$)$.

Change in BMI z-score:

Intervention: -0.05

Control group: -0.01

Adjusted BMI difference between intervention and control:

-0.2 (95\%Cl: -0.78 to 0.39$), p=0.5$

BMI (difference between intervention and control in change between groups):

Girls: -0.05 ( $95 \% \mathrm{Cl}$ : 0.18 to 0.08 ) 
Table 3 Summary of findings from included studies (Continued)

\begin{tabular}{|c|c|c|c|}
\hline $\begin{array}{l}\text { Author, Year, Country, } \\
\text { Name of Cohort/ study, }\end{array}$ & $\begin{array}{l}\text { Study design, Participant } \\
\text { characteristics }^{\mathrm{a}}\end{array}$ & Key Intervention characteristics & $\begin{array}{l}\text { Main findings and results for BMI/BMI z- } \\
\text { score outcomes }{ }^{b}\end{array}$ \\
\hline
\end{tabular}

Citation

$\begin{array}{ll}\text { Intervention in } & \text { Total } n=978 \\ \text { Teenagers) } & \text { Follow up immediately after intervention } \\ & \text { - Teacher training: Teachers received a manual to support } \\ & \text { them in delivering the intervention } \\ & \text { - Digital component: Individual advice provided by CD rom } \\ & \text { - Parental involvement: None reported } \\ & \text { - Behaviour change theory: Not specified }\end{array}$

\section{Medium Risk of Bias}

Ermetici, 2016 [37], Italy ${ }^{c} \quad$ Non-randomised quasiEAT Study

$\begin{array}{ll} & \text { Mean age 12.5 (0.4) } \\ & \text { Intervention: } n=262 \\ & \text { Control: } n=225 \\ & \\ \text { Wilksh, 2015, Australia } \quad & \text { Four arm RCT with mu } \\ \text { [62] } & \text { educational modules } \\ & \text { Mean age: 13.21 (0.68) } \\ & \text { Media Smart (MS): } N= \\ & \text { Life Smart (LS): } N=347 \\ & \text { HELPP (HP): } N=225 \\ & \text { Control (C): } N=473 \\ & \\ & \\ & \text { Two armed quasi- } \\ & \text { experimental study } \\ & \text { Mean age: } 13.3(0.79) \\ \text { Lazorick, 2015 [41], US } & \text { Intervention: } n=189 \\ \text { The MATCH Intervention } & \text { Control: } n=173\end{array}$

Grydeland, 2014 [40], Norway ${ }^{d}$

HEIA Intervention Study
Two armed RCT Mean age $=11.2(0.3)$

Intervention: $n=465$ Control: $n=859$
Bonsergent, 2013 [35], France PRALIMAP

Fairclough, 2013 [38], UK

The CHANGE!

Intervention

Williamson, 2012 [46] USA
- Primary outcome: BMI Z-score

- Intervention duration: 2 school years

- Follow up after intervention: Immediate post intervention

- Teacher training: Text book to aid lessons

- Digital component: Automated text messages

- Parental involvement: Text messages

- Behaviour change theory: not specified

- Primary outcome: risk of eating disorders

- Duration of intervention (LifeSmart): 5 weeks

- Follow up after intervention: post program, 6 month and 12 months

- Teacher training: None reported

- Digital component: None reported

- Parental involvement: None reported

- Behaviour change theory: not specified

- Primary outcome: BMI and BMI Z-score

- Intervention duration: 14 weeks

- Follow up after intervention: post intervention and one

year

- Teacher training: One day of teacher training provided and a two-day orientation

- Digital component: None reported

- Parental involvement: None reported

- Behaviour change theory: Social cognitive and selfdetermination theory

- Primary outcome: BMI and BMI Z-score

- Intervention duration: 20 months

- Follow up after intervention: immediate post intervention

- Teacher training: PE teachers were enrolled in a course

- Digital component: Computer tailored individual advice

- Parental involvement: Parent-based fact sheets

- Behaviour change theory: socio-ecological framework

Boys: $-0.02(95 \% \mathrm{Cl}-0.11$ to 0.16$)$

After 2 years, BMI z-score (adjusted difference): 0.18 (95\%Cl: -0.27 to -0.09$), p=0.003$

Group by time effect showed no significant effects on BMI for boys or girls at 12 month follow up.

Mean change BMI z-score immediately post intervention

Intervention: -0.06 (95\%Cl: -0.08 to -0.03$)$

Control: 0.02 (95\%Cl: -0.004 to 0.05 )

$p$-value for difference $<0.001$

Post intervention BMl z-score (adjusted for baseline values)

Girls

Intervention: $-0.8(95 \% \mathrm{Cl}:-0.14,-0.02)$

Control: 0.03 (95\%Cl: $-0.01,0.08)$

$\mathrm{p}$-value for difference $=0.003$

Boys

Intervention: $-0.01(-0.07,0.05)$

Control: -0.05 (95\%Cl: $-0.09,-0.00)$

$\mathrm{p}$-value for difference $=0.32$

Total

Intervention: - 0.04 (95\%Cl: - 0.09, 0.00)

Control: -0.01 (95\%Cl: $-0.04,0.02)$

$\mathrm{p}$-value for difference $=0.227$

$\mathrm{RCT}$

Mean age $15.6(0.7)$

Intervention: 3424

Control: 2947

RCT

Mean age (control group):

$10.7(0.3)$

Control: 117

Intervention: 89

RCT

Mean age: $12.9(1.2)$

Intervention: $\mathrm{PP}=511$; PP+

$\mathrm{SP}=516$

Control: 307
- Primary outcomes: BMI and BMI Z-score

- Duration of intervention: 24 months

- Follow up: at 12 months and immediately post

intervention

- Teacher training: None reported

- Digital component: None reported

- Parental involvement: None reported

- Behaviour change theory: None reported

- Primary outcomes: Waist circumference, BMI and BMI z-

score

- Duration of intervention: 20 weeks

- Follow up at immediately post intervention (20 weeks) and 30 weeks

- Teacher training: $4 \mathrm{~h}$ in how to deliver the curriculum

- Digital component: CD ROM

- Parental involvement: None reported

- Behaviour change theory: Social cognitive theory

- Primary outcomes: Percentage body fat and BMI z-score

- Duration of intervention: 28 months.

- Follow up at 18 months and 28 months (immediately

post intervention).

- Teacher training: None reported

- Digital component: Online platform

- Parental involvement: Emails to parents.
BMI z-score change at 12 months post intervention:

$\beta=0.004(95 \% \mathrm{Cl}:-0.026,0.034)$

BMI z-score adjusted change at 20 weeks: $\beta=-0.04$ (95\% Cl: $-0.22,0.15), p=0.68$ BMI z-score adjusted change at 30 weeks: $\beta=-0.24$ (95\% Cl: $-0.48,-0.003), p=0.04$

Adjusted difference between control and intervention BMl z-scores at 28 months: Boys: $\beta=-0.034$

Girls: $\beta=-0.035$ 
Table 3 Summary of findings from included studies (Continued)

\begin{tabular}{|c|c|c|}
\hline $\begin{array}{l}\text { Author, Year, Country, } \\
\text { Name of Cohort/ study, } \\
\text { Citation }\end{array}$ & $\begin{array}{l}\text { Study design, Participant } \\
\text { characteristics }^{\mathrm{a}}\end{array}$ & Key Intervention characteristics \\
\hline & & - Behaviour change theory: Not reported. \\
\hline $\begin{array}{l}\text { Neumark-Sztainer, } 2010 \\
\text { [55], US } \\
\text { New Moves }\end{array}$ & $\begin{array}{l}\text { RCT } \\
\text { Mean age: } 15.8(1.2) \\
\text { Intervention: } 182 \\
\text { Control: } 174 \\
\text { Girls only }\end{array}$ & $\begin{array}{l}\text { - Primary outcomes: Physical activity levels } \\
\text { - Duration of intervention: one school year. } \\
\text { - Follow-up at immediately post intervention and } 9 \text { months } \\
\text { post intervention. } \\
\text { - Teacher training: None reported. } \\
\text { - Digital component: None reported. } \\
\text { - Parental involvement: Parent outreach and parent- } \\
\text { daughter retreat days. } \\
\text { - Behaviour change theory: Social cognitive theory. }\end{array}$ \\
\hline $\begin{array}{l}\text { Mihas, } 2009 \text { [54], Greece }{ }^{c} \\
\text { VYRONAS study }\end{array}$ & $\begin{array}{l}\text { RCT } \\
\text { Mean age (control) } 13.3 \\
\text { (0.9) } \\
\text { Intervention: } n=98 \\
\text { Control: } n=93\end{array}$ & $\begin{array}{l}\text { - Primary outcomes: dietary habits and BMI } \\
\text { - Duration of intervention: } 12 \text { weeks } \\
\text { - Follow ups after intervention: } 15 \text { days and } 12 \text { months } \\
\text { - Teacher training: materials and two 3-h seminars } \\
\text { - Digital component: none reported } \\
\text { - Parental involvement: nutrition education and behaviour } \\
\text { change } \\
\text { - Behaviour change theory: social learning theory }\end{array}$ \\
\hline Young, 2006 [63], USA & $\begin{array}{l}\text { RCT } \\
\text { Mean age } 13.8(0.5) \\
\text { Intervention: } n=111 \\
\text { Control: } n=99 \\
\text { Girls only }\end{array}$ & $\begin{array}{l}\text { - Primary outcomes: physical activity and markers of } \\
\text { cardiovascular disease risk factors } \\
\text { - Duration of intervention: } 8 \text { months } \\
\text { - Follow up immediately after intervention } \\
\text { - Teacher training: not needed as intervention delivered by } \\
\text { research staff } \\
\text { - Digital component: none reported } \\
\text { - Parental involvement: family workshops, monthly } \\
\text { newsletters and parent/child homework } \\
\text { - Behaviour change theory: social action theory }\end{array}$ \\
\hline
\end{tabular}

\section{High risk of Bias}

Benitez-Andrades (2020), ${ }^{\mathrm{d}}$ [65] Spain
Non-randomised pre-post quasi experimental study design with control group Mean age: 12.8 for $C$ and 12.6 for 1

Intervention: $n=139$

Control: $n=91$
- Primary outcome: BMI age-adjusted percentile, physical activity, eating habits

- Duration of intervention: 14 weeks

- Follow up: immediately after intervention only

- Delivered by researchers

- Digital component: Facebook-based intervention

- Parental involvement: None

- Behaviour Change Theory: Not specified
Main findings and results for BMI/BMI zscore outcomes ${ }^{b}$

No change in BMl at 15 days Mean BMI (adjusted) at 12 months vs baseline: Int: 23.3 (SD 2.8) vs 24.0 (SD 3.1) $p<0.001$ Con: 24.8 (SD 3.8) vs 24.3 (SD 3.3) $p=0.36$

Adjusted mean BMI change: Intervention: 0.3 (SE 0.2) $p=0.2$ Control: 0.2 (SE 0.2) $p=0.34$ Between group $p=0.81$
Adjusted BMI difference between groups at 9 month post intervention follow-up:

Intervention group:

BMI age-adjusted percentile

( $\geq 50$ initial i.e. overweight)

Mean Pre: 77.59

Mean post: 72.85

Z: -5.394

$p=0.000$

Control group:

BMl age-adjusted percentile

$(\geq 50$ initial)

Mean pre: 78.09

Mean post: 77.49

$\mathrm{Z}=0.241$

$p=0.809$

Non-adjusted mean difference in BMI between intervention and control: 1.9 (95\% Cl: 0.035, 3.76)

Change in prevalence for overweight: $-0.8 \%$

Froberg, 2018 [64], Sweden with control group

Mean age: $12.8(0.5)$ Intervention: 51 Control: 47

- Primary outcome: physical activity, food habits, and behaviour change

- Duration of intervention: 2 years

- Follow up: 2 years and 4 months from after baseline

- Delivered by researchers

- Digital component: Facebook group for communication between researchers and students, however main intervention was delivered in class.

- Parental involvement: None

- Behaviour change theory: empowerment-based health promotion, shared decision making

Busch, 2015 [49], The Netherlands ${ }^{\mathrm{d}}$

Utrecht Healthy School (UHS)

Millar, 2011 [43], USA c
Non-randomised controlled trial

Age group: high school students 1 year $(N=969)$

2 years $(N=605){ }^{a}$

Quasi-experimental study Mean age: $14.6(1.42)$ Intervention: $n=1276$ Control: 778
- Primary outcome: BMI, health behaviour and psychosocial health

- Intervention duration: The UHS was integrated into the school curriculum for 2 years

- Follow up after intervention: 1 and 2 years from baseline

- Teacher training: For teachers and head teachers

- Digital component: None reported

- Parental involvement: participation in school projects

- Behaviour change theory: not specified

- Primary outcomes: BMI, BMI z-score and body composition

- Duration of intervention: Not reported

- Follow up at (m; SD) 2.3 (0.68) years from baseline.

- Teacher training: CPD for PE teachers.

- Digital component: None reported.

- Parental involvement: parent information, family and
Adjusted BMI change from baseline for interventions schools compared with control schools

School A

Year 1: $\beta=-0.48(p<0.05)$

Year 2 : $\beta=-0.58(p<0.05)$

School B

Year 1: $\beta=-0.05(p>0.05)$

Year 2 : $\beta=-0.43(p>0.05)$

Adjusted difference between intervention and control BMI z-scores at follow-up:

-0.07 (SE 0.03), $p=0.03$ 
Table 3 Summary of findings from included studies (Continued)

\begin{tabular}{|c|c|c|c|}
\hline $\begin{array}{l}\text { Author, Year, Country, } \\
\text { Name of Cohort/ study, } \\
\text { Citation }\end{array}$ & $\begin{array}{l}\text { Study design, Participant } \\
\text { characteristics }^{\mathrm{a}}\end{array}$ & Key Intervention characteristics & $\begin{array}{l}\text { Main findings and results for BMI/BMI z- } \\
\text { score outcomes } b\end{array}$ \\
\hline & & $\begin{array}{l}\text { home environment } \\
\text {. Behaviour change theory: Not reported. }\end{array}$ & \\
\hline $\begin{array}{l}\text { Graham, } 2008 \text { [51] } \\
\text { Schneider, } 2007 \text { [69] } \\
\text { USA }\end{array}$ & $\begin{array}{l}\text { Non-randomised controlled } \\
\text { trial } \\
\text { Mean age } 15.04(0.79) \\
\text { Intervention: } n=63 \\
\text { Control: } n=59 \\
\text { Girls only }\end{array}$ & $\begin{array}{l}\text { - Primary outcomes: Cardiovascular fitness and physical } \\
\text { activity levels } \\
\text { - Duration of intervention: } 9 \text { months } \\
\text { - Follow up after immediately after intervention } \\
\text { - Teacher training: No teacher training described, unclear } \\
\text { who delivered the intervention } \\
\text { - Digital component: None reported } \\
\text { - Parental involvement: None reported } \\
\text { - Behaviour change theory: not specified }\end{array}$ & $\begin{array}{l}\text { No significant difference }(p=0.1) \text { between } \\
\text { groups in change in BMl percentile }\end{array}$ \\
\hline $\begin{array}{l}\text { Webber, } 2008 \text { [61], US } \\
\text { Trial of Activity for } \\
\text { Adolescent Girls } \\
\text { (TAAG) }\end{array}$ & $\begin{array}{l}\text { Cluster RCT } \\
\text { Mean ages: } \\
\text { 6th graders } 12.0 \\
\text { 8th graders } 14.0 \\
n=1721 \text { (6th grade 2003) } \\
n=3504 \text { (8th grade 2005) } \\
n=3502 \text { (8th grade 2006) } \\
\text { Girls only }\end{array}$ & $\begin{array}{l}\text { - Primary outcomes: physical activity levels and body } \\
\text { composition measurements } \\
\text { - Duration of intervention: } 2-3 \text { years } \\
\text { - Follow-ups: } 2 \text { and } 3 \text { years post baseline } \\
\text { - Teacher training: PE teachers and Program Champions } \\
\text { were trained by TAAG investigators } \\
\text { - Digital component: None reported } \\
\text { - Parental involvement: None reported } \\
\text { - Behaviour change theories: operant learning theory, social } \\
\text { cognitive theory, organizational change theory, and } \\
\text { diffusion of innovation model }\end{array}$ & $\begin{array}{l}\text { BMI mean difference: } \\
\text { 6th grade (2003): }-0.2 \\
\text { (95\%Cl: }-1.0 \text { to } 0.6) \\
\text { 8th grade (2005): }-0.2 \\
\text { (95\%Cl: }-0.6 \text { to } 0.2 \text { ) } \\
\text { 8th grade (2006): } 0.1 \\
\text { (95\%Cl: }-0.4 \text { to } 0.7 \text { ) }\end{array}$ \\
\hline $\begin{array}{l}\text { Foster, } 2008 \text { [39], } \\
\text { US'(SNIP study (School } \\
\text { Nutrition Policy } \\
\text { Initiative) }\end{array}$ & $\begin{array}{l}\text { RCT } \\
\text { Mean age (control) } 11.2 \\
\text { (1.0) } \\
\text { Intervention: } n=749 \\
\text { Control: } n=600\end{array}$ & $\begin{array}{l}\text { - Primary outcomes: incidence of overweight and obesity } \\
\text { - Duration of intervention: } 2 \text { years } \\
\text { - Follow-up immediately after intervention } \\
\text { - Teacher training: Teachers were offered up to } 10 \mathrm{~h} \text { of } \\
\text { training per year } \\
\text { - Digital component: None reported } \\
\text { - Parental involvement: Parent outreach } \\
\text { - Behaviour change theory: Not specified }\end{array}$ & $\begin{array}{l}\text { BMI (adjusted difference): } \\
-0.04 \text { ( } 95 \% \text { Cl: }-0.27 \text { to } 0.19), p=0.71 \\
\text { BMI z-score (adjusted difference): } \\
\text { - } 0.01 \text { ( } 95 \% \text { Cl: }-0.08 \text { to } 0.06 \text { ), } p=0.80 \\
\text { Predicted odds ratio for incidence of overweight } \\
\text { (adjusted): } 0.67 \text { ( } 95 \% \text { Cl: } 0.47 \text { to } 0.96 \text { ) } p<0.05\end{array}$ \\
\hline $\begin{array}{l}\text { Rosenbaum, } 2007[58] \\
\text { USA c }\end{array}$ & $\begin{array}{l}\text { RCT } \\
\text { Mean age (control) } 13.6 \\
(0.2) \\
\text { Intervention: } n=49 \\
\text { Control: } n=24\end{array}$ & $\begin{array}{l}\text { - Primary outcomes: Markers of insulin sensitivity and } \\
\text { inflammation } \\
\text { - Duration of intervention: } 3-4 \text { months } \\
\text { - Follow up immediately after intervention } \\
\text { - Teacher training: None (delivered by researchers) } \\
\text { - Digital component: None reported } \\
\text { - Parental involvement: None reported } \\
\text { Behaviour change theory: Not specified }\end{array}$ & $\begin{array}{l}\text { BMl at baseline and follow-up: } \\
\text { Control: } 24.3 \text { (SD: } 1.8) \text { to } 24.8 \text { (SD: 1.9) } \\
p \geq 0.05 \\
\text { Intervention: } 24.7 \text { (SD: 1.4) to } 24.0 \text { (SD:1.5) } \\
p<0.05\end{array}$ \\
\hline $\begin{array}{l}\text { Amaro, } 2006 \text { [34], Italy, } \\
\text { Kaledo pilot (See } \\
\text { Viggiano, } 2015 \text { [45]) }\end{array}$ & $\begin{array}{l}\text { RCT } \\
\text { Mean age (control) } 12.5 \\
\text { (0.7) } \\
\text { Intervention: } n=153 \\
\text { Control: } n=88\end{array}$ & $\begin{array}{l}\text { - Primary outcomes: dietary behaviours and BMI Z-score } \\
\text { - Duration of intervention: } 24 \text { weeks } \\
\text { - Follow up: immediately after intervention } \\
\text { - Teacher training: teachers trained in playing the game. } \\
\text { - Digital component: None reported } \\
\text { - Parental involvement: None reported } \\
\text { - Behaviour change theory: Not specified }\end{array}$ & $\begin{array}{l}\text { BMI z-score (adjusted mean controlling for base- } \\
\text { line values): } \\
\text { Intervention: } 0.35 \text { ( } 95 \mathrm{Cl} \%: 0.30 \text { to } 0.39 \text { ) } \\
\text { Control: } 0.41 \text { ( } 95 \% \text { Cl: } 0.35 \text { to } 0.47 \text { ) }\end{array}$ \\
\hline
\end{tabular}

${ }^{a}$ Some studies only reported age by group or group and sex, but were similar in both groups; Where sample size for intervention and control groups are not reported, total size is presented

${ }^{d}$ Significant effect in a subset analysis

61 to +11 [47]. As most studies were multi-component, developing a narrative summary with exclusive groups based on characteristics was not feasible. For discussion, we present groups based on their dominant intervention components.

\section{Mode of intervention delivery}

Most interventions were delivered by teachers $(n=22)$ [34-42, 45, 46, 48-50, 52-54, 57, 59, 61, 62] followed by researchers $[35,55,56,58,60,63-65]$, school nurses $[44,47]$ and in others a school project officer and physical education teacher [43] or project managers [66].
The study by Bogart et al. trained 'peer leaders', in addition to teachers, to promote and model healthy behaviours and engage other students [48, 70]. Nineteen studies reported training the teachers to deliver the intervention through a variety of means including workbooks or other training materials and face-to-face sessions [34, 36-43, 45, 48-50, 52-54, 57, 59, 61]. For example, the Health in Adolescents (HEIA) Intervention [40] included two courses in physical education based on a previously validated programme for teachers [71]. Millar et al. provided continuing professional development (CPD) programmes for physical education teachers 


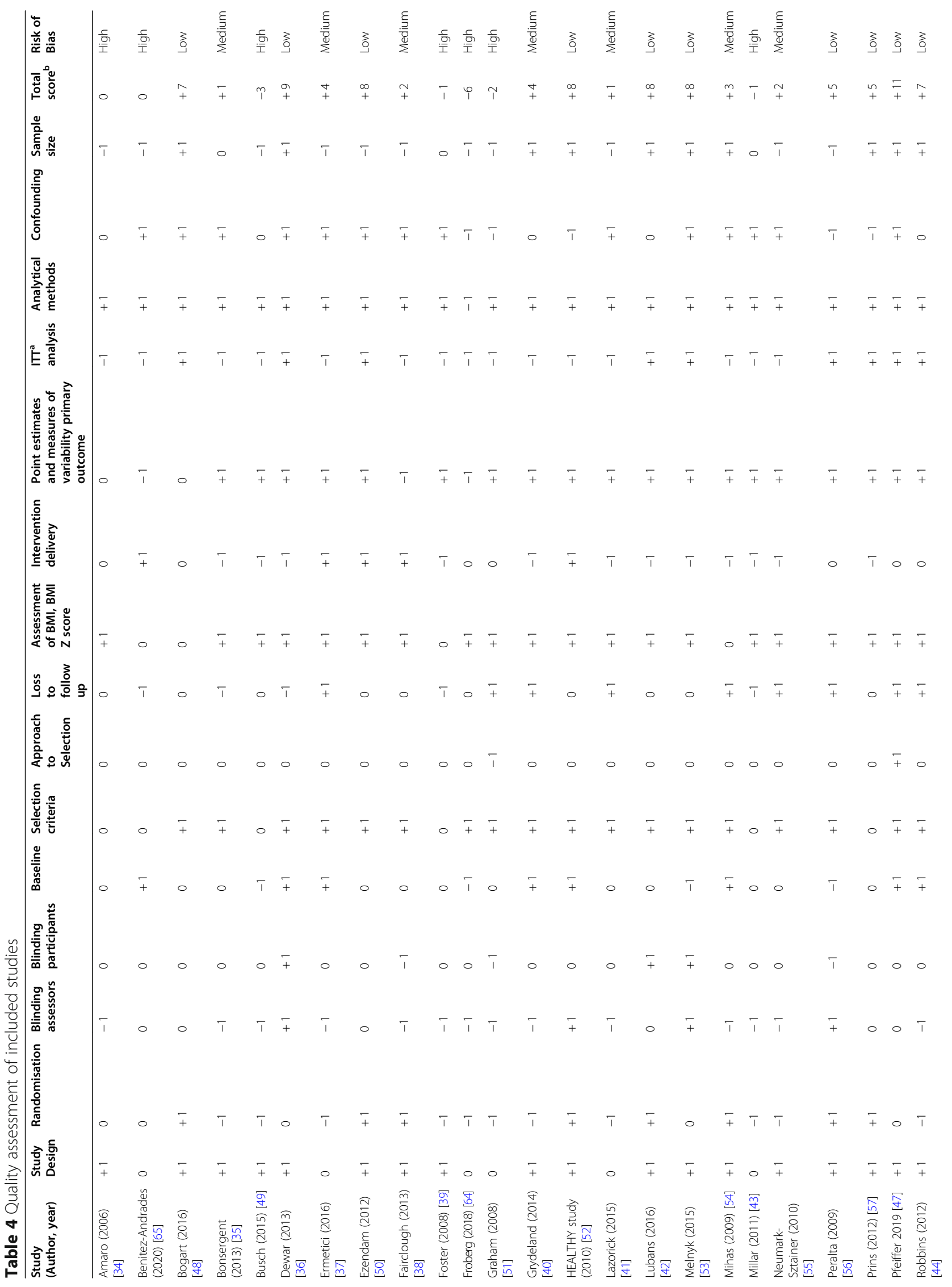




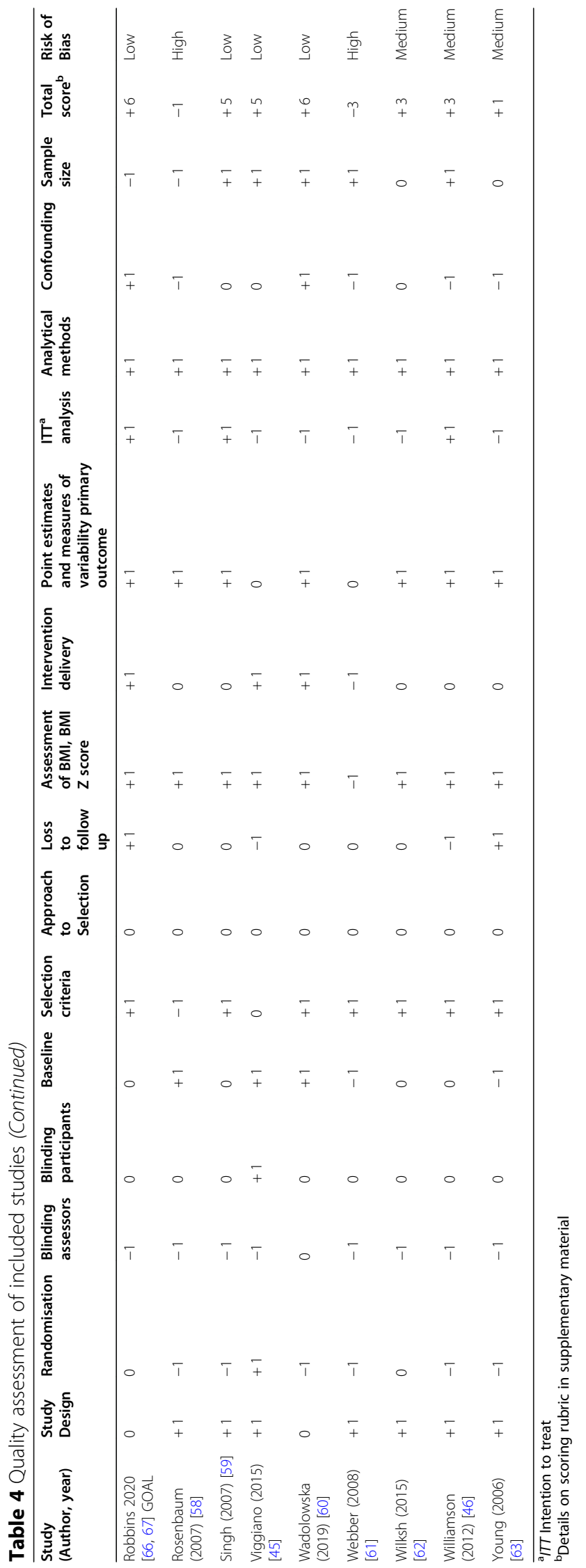


[43]. CPD was defined as the skills, knowledge and experience gained by teachers beyond any initial formal or informal training.

\section{Parental involvement}

Sixteen interventions had parental involvement [36, 37, $39,40,42,43,46,48,49,52-56,63,66]$. The modes of parental involvement are summarized in Tables 2 and 3. Busch et al. conducted an educational intervention integrated into the regular curriculum [49]. The study by Mihas et al. aimed to improve knowledge, behavioural capability, expectations and self-efficacy [54]. Parents were also encouraged to improve their own dietary behaviours. Grydeland et al. collaborated with school principals, teachers, school health services and parent committees [40]. Teachers delivered the lessons and handed out monthly factsheets to parents and an activity box to students with sports equipment and toys to promote physical activity. Parents in the study conducted by Bogart et al., also engaged in homework activities with the students, which included completing worksheets on food preferences among different members of the family and types of fruits and vegetables kept at home [48]. Finally, three of the studies only provided information to parents [37, 43, 53]. Parental involvement was a key component of the Guys/ Girls Opt for Activities for Life (GOAL) intervention [66] which included parentadolescent dyads for group meetings targeting selfefficacy, social support and motivation. The meetings aimed to assist parents in supporting students' physical activity and healthy eating habits through discussion on behavioural strategies as well as healthy cooking sessions.

\section{Digital interventions}

Sixteen studies used digital media such as apps, websites, CD-ROMs and computer-tailored information [35-38, $40,42,43,46,50,55,59,63-66]$. The HEIA intervention included the provision of information to increase awareness of recommended physical activity levels and fruit and vegetable consumption [40]. They also provided tailored advice to students (a subgroup within the intervention) on how to change dietary habits, screen time and physical activity levels. An intervention in Italian schools included 16 health-promoting lessons (delivered by nutritionists) along with text messages for daily exercise and diet advice, as well as environmental modification (e.g. vending machines for healthy food items) [37]. This quasi-experimental study evaluated an intervention in which automated text messages were sent to students and parents three times a week, close to mealtimes, to promote discussions in the family related to healthy eating habits. The FATaintPHAT intervention consisted of a computer-tailored intervention to help prevent excessive weight gain by improving diet, reducing sedentary behaviours and increasing physical activity, with additional modules on weight management [50]. The modules also included specific goal-setting and action planning with normative and comparative (with peers) feedback. Three studies also used social media (such as Facebook) to provide a platform for communication with the researcher (who delivered the intervention) [64], information on sessions [64], and weekly Facebook participation for parents in the GOAL programme [66]. Another intervention [65] used a social-network based eHealth intervention to improve diet and physical activity habits. The participants could use the social network platform to develop friendships and interact with each other while receiving information about nutrition and physical activity. They were also given rewards for improving their habits.

\section{Change in environment}

Ten studies included measures to improve the environment, in addition to educational components, or school policy change to increase accessibility and/or improve facilities in schools for sports, social marketing and providing healthier meals [37, 39-41, 43, 49, 52, 53, 58, 70]. Three studies also applied environmental modification in neighborhoods through community activities or providing information on improving home/neighborhood environment $[35,49,59]$.

Millar et al. provided a school-based intervention with a community component focusing on promoting healthy breakfasts, increasing fruit and vegetable consumption, and improving school meals [43]. Rosenbaum et al. delivered an intervention with the primary aim of reducing risk of Type 2 diabetes in adolescents consisting of health, nutrition and exercise classes, a programme on diabetes risk along with 45-min sessions on Type 2 diabetes prevention in class [58]. The MATCH intervention [41] and the COPE program [53] also used additional physical activity sessions to support the educational interventions. COPE was a manualized 15 -session educational and cognitive behavioural skills building programme that also aimed to prevent symptoms of depression in adolescents. The EAT project included school environmental changes such as providing healthier snacks in vending machines, placing educational posters throughout the school, and creating additional play areas [37]. Bogart et al. also used additional food environment modification strategies by providing a variety of fruits and vegetables during school lunch and free water [48]. Campaigns were also conducted to disseminate the messages widely through the schools. Two studies included lunch sessions or breaks to provide healthy foods such as fruit and vegetables [40,55], and two trialed a modified school nutrition policy $[39,49]$. 


\section{Meta-analysis}

Of the 33 studies included in the review, 14 studies reporting outcomes based on BMI z-score were included in the meta-analysis [34-47]. There were too few studies that reported effect sizes in a consistent manner to conduct further meta-analyses. There was a high level of heterogeneity between the studies $\left(\mathrm{I}^{2}=62.7 \%\right)$, as seen in Fig. 2. The overall pooled estimate of change in BMI zscore in the intervention group, compared with the control group, was $-0.06,95 \%$ CI $(-0.10,-0.03) ; p<0.001$.

We constructed a funnel plot using the mean difference and standard error of the mean difference in BMI to assess the risk of publication bias (Fig. 3). The asymmetry in the plot indicates a degree of bias within this subset of 14 studies included in the meta-analysis, which might have led to overestimation of effect size. The findings discussed in the meta-analysis and narrative synthesis should therefore be interpreted with caution due to the likelihood of publication bias, high heterogeneity and small effect size.

\section{Key features of studies with significant effect on BMI outcomes}

Overall, fourteen studies reported a significant reduction in BMI and/or BMI z-score [37-41, 43, 45, 48, 49, 52$54,58,65]$. Of these, four studies reported significant effects in only a subset of the population (See Table 3) $[40,48,49,65]$. All effective studies with a significant effect on BMI outcomes had a face-to-face component for intervention delivery in the classroom, except one, which was only digital [65]. Of the 16 studies that included parents, eight reported significant effects on BMI outcomes [37, 39, 40, 43, 49, 52-54]. Seven of the effective interventions were RCTs [38-40, 45, 48, 52-54] and seven were non-RCTs [37, 41, 43, 49, 58, 65]. One quasi-experimental study used a capacity-building approach with a community-based component to promote healthy eating and physical activity [43]. There was high variation in the duration of the interventions, from 12 weeks [54] to 3 years [43], with four studies in which the intervention was delivered for a year or more [37, 39, 43, 49]. Even though 14 studies showed significant effect on BMI outcomes, only four studies had a low risk of bias $[45,48,52,53]$. Five studies had a high risk $[39,43,49$, $58,65]$, and the rest a medium risk [37, 38, 40, 41, 54], and this could affect the reliability of the findings. Some studies that were of a low risk of bias did not show significant effects of the intervention on BMI outcomes $[36,42,44,47]$.

Of the 22 interventions delivered by teachers, twelve showed significant effects on BMI outcomes [37-41, 43, 45, 48, 49, 52-54]. Providing CPD/training for teachers prior to the intervention, including some form of faceto-face sessions such as workshops and seminars, was a feature of effective interventions. In the Change! intervention, Fairclough et al. developed the curriculum and resources through formative work with teachers, parents and children [38]. This intervention focused on the interaction between social and environmental factors and their effect on behaviour and provided education on physical activity and nutrition. Subgroup analysis revealing that post-intervention (20 weeks) effects on BMI were significantly greater in girls, but effects on BMI were not sustained at 30 weeks. In the Kaledo study, teachers were trained in how to facilitate and supervise students while playing the game (Kaledo) and there were sustained significant reductions in BMI z-score at 6 and 18-month post-intervention [45]. The game was personalized (participants entered their BMI values) and included a 'punishment and reward system' for specific dietary behaviours. It aimed to improve nutrition knowledge and influence dietary habits and eating behaviours of adolescents. Three studies provided more intensive CPD for at least 1 day $[39,41,53]$, with one study providing 10 one-hour training sessions across the whole year [39].

Five interventions based on the on social cognitive theory found a statistically significant effect on BMI outcomes [38, 41, 52-54]. The HEALTHY study was a multi-component study which included parents, environmental change, homework activities during breaks and interactive educational lessons in class called 'FLAS $\mathrm{H}^{\prime}$ delivered by teachers [52]. The module targeted awareness, knowledge, behavioural skills such as goalsetting and peer influence. However, the intervention was delivered in a school with high proportion of ethnic minorities who were at a risk of diabetes $(54.2 \%$ Hispanic, $18 \%$ black). The study led to a non-significant decrease in prevalence of overweight and obesity in both intervention and control schools. Additionally, the mean BMI z-score was significantly lower in the intervention schools than in the control school. The effect of the intervention was higher among students with obesity. However, two multi-component interventions with low risk of bias (NEAT girls and ATLAS) were also based on social cognitive theory and had no short or long-term effects on BMI. These interventions included sports sessions, seminars and nutrition workshops, parent newsletters, pedometers and text messages [36, 42]. Also, of the 16 interventions that included parental involvement, 8 found significant outcomes on BMI [37, $39,40,43,48,52-54]$. The mode of parental involvement in the effective interventions included parent newsletters [52, 53] homework with parents [48], text messages [37], nutrition education and behaviour change for parents [40, 43, 54]. A quasi experimental study [65] led to significant improvement in a subgroup with initial BMI age-adjusted percentile $>50 \%$. Interestingly, there 


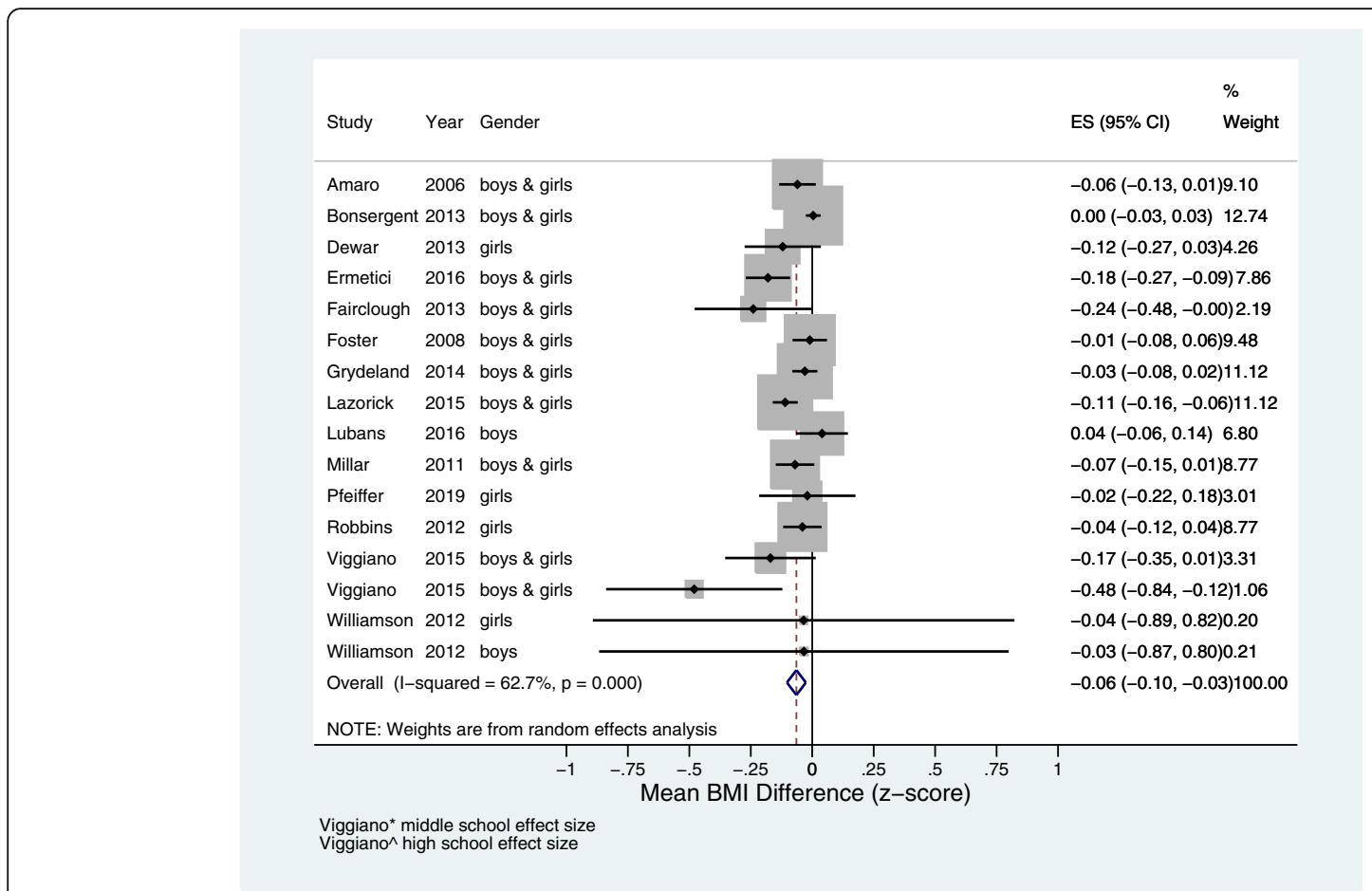

Fig. 2 Meta-analysis of 14 studies showing the effect of educational interventions on BMI z-score. The pooled estimate shows significant difference between intervention and control in change in BMI z-score

was also a significant increase in BMI age-adjusted percentile for students with BMI percentile less than $50 \%$ at baseline. The intervention used methods such as developing peer networks and rewarding good practices to encourage adolescents make healthier choices.

\section{Discussion}

The purpose of this review was to synthesize evidence regarding the effectiveness of school-based health education programmes in reducing BMI and preventing overweight and obesity in adolescents. Overall, we found

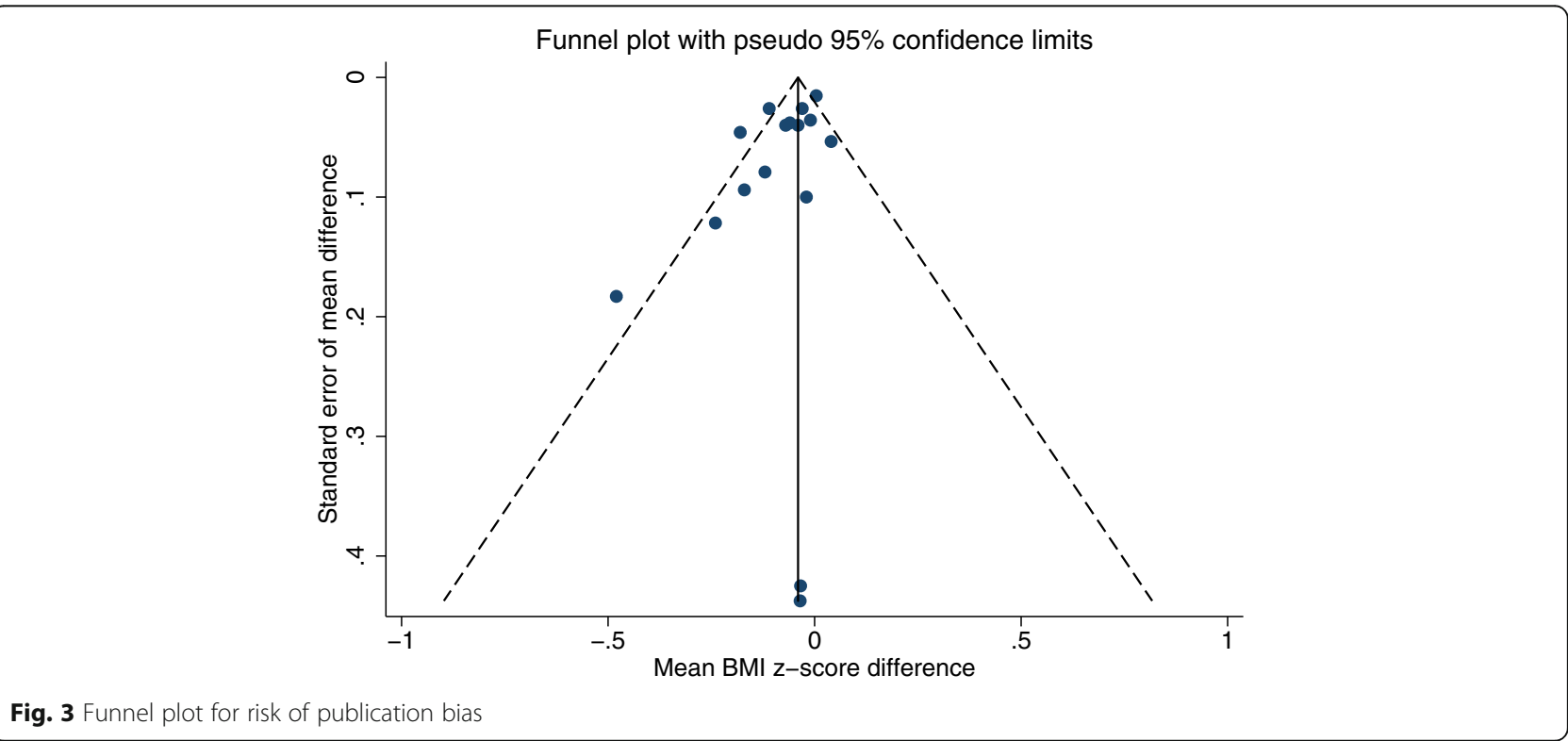

Fig. 3 Funnel plot for risk of publication bias 
small but significant reductions in BMI z-score. All but two $[58,65]$ of the effective interventions were delivered by teachers who were trained prior to the intervention, suggesting that though school-based interventions are often delivered through school-staff, appropriate training/ CPD prior to the intervention could be a crucial component to support the provision and uptake of the intervention. Similarly, many of the effective interventions had included parental involvement and modifications to the school environment. The studies in this review mainly evaluated multi-component interventions that used health education as a tool to improve health behaviours related to diet, physical activity and body composition measures.

Schools are a commonly-used setting for behavioural interventions for preventing obesity and overweight and improving diet and physical activity levels in children and adolescents, as they provide an easy channel for accessing this age group. However, previous reviews of school-based interventions have shown mixed results for BMI outcomes [72, 73]. These reviews suggest that, for children and adolescents, effective interventions targeted direct physical activity and weight reduction through physical education programmes combined with nutrition education. Some studies have shown improvements in the prevalence of overweight and obesity within this age group but only modest effects on BMI [23, 74]. Dietz and Gortmaker (2001) developed a logic model for schools describing the range of factors that influence the energy balance in students [75]. These included a coordinated school health programme that promoted healthy diets and physical activity, school policies and physical education sessions in schools and the surrounding community, along with environmental factors. The results of the present review suggest that schools have the opportunity to effectively deliver evidence-based interventions to prevent obesity.

Various creative methods have been used to engage adolescents in the studies in this review, such as board games [45], digital components (online counselling, SMS messaging) $[36,37,40,55,57,59,65,66]$ and retreat days [55]. A recent systematic review evaluated the effectiveness of digital interventions in improving diet quality and increasing physical activity in adolescents, suggesting that significant behaviour change can be achieved when health education, goal setting, selfmonitoring and parental involvement are included (mainly using web-based platforms, followed by text messages, and games) [76]. Computer-based nutrition education has also led to short-term improvement in BMI [77].

The success of interventions with health education also depends on how the messages are delivered [78]. Complex interventions that are more engaging for adolescents should be developed based on user preferences [79]. Recent RCTs such as the LifeLab intervention have focused on improving adolescents' understanding of the science behind health messages and motivating them to improve their diet and physical activity levels through hands-on engagement with science [80]. This complex intervention also aims to improve health literacy in adolescents, with preliminary results showing an improvement in their knowledge about risks of NCDs.

It must be noted that many of the studies included in this review were effective in improving other outcomes such as diet, physical activity levels and body fat percentage $[34,37,44-46,51,53,57,58]$. For example, three studies with no effects on BMI significantly improved levels of moderate to vigorous physical activity [44, 51, 57], and another led to reduced body fat percentage [46]. Four studies with no effect on BMI recruited teenagers with low levels of activity [36, 44, 51, 56] or low cardiorespiratory fitness [56] at baseline, which could have affected the uptake of the intervention. Similarly, some interventions led to a significant effect on BMI for adolescents with obesity at baseline $[48,52,53]$. This could potentially be due to differences in physiological responses to weight loss interventions between adolescents with obesity and those with normal BMI [81]. It should be noted as well that, although we have excluded studies focusing on adolescent eating disorders, future interventions should consider potential unintended effects on body image, eating disorders and other psychological attributes [82].

\section{Role of stakeholders}

Effective interventions often included key stakeholders such as teachers and parents. Previous studies related to other issues in adolescence such as consequences of alcohol consumption have shown that students preferred interventions delivered by teachers $[83,84]$ and thus the teacher-student relationship can support the effectiveness of school-based interventions. However, these findings should be interpreted with caution. It was not possible to perform a subgroups analysis to determine the role of key stakeholders in the included studies.

Previous systematic reviews in this area reached similar conclusions; that effective physical activity-based interventions, resulting in improved BMI outcomes, were characterized by familial involvement and training for teachers and students on behavioural techniques such as self-monitoring [73]. In this review, most of the effective studies were facilitated by teachers who received CPD in a face-to-face format. Behaviour change frameworks have highlighted the importance of 'facilitators' (e.g. qualifications and experience of those delivering the intervention) and 'pedagogy' (teaching strategies used by 
facilitators to deliver the intervention components effectively) in improving intervention engagement and outcomes [85]. A systematic review of teacher CPD in school-based physical activity interventions showed that such programmes were beneficial, particularly when they were conducted for more than 1 day, provided comprehensive pedagogy content, were framed by a theoretical model and measured teachers' satisfaction with training and content [19]. During data extraction, the reviewers noted that details of teacher CPD are often not elaborated upon or even reported in studies, and hence may have been missed by the present review. The 'It's your move!' project, which used peer-led approaches and capacity-building for teachers, schools and parents highlighted certain challenges such as making time for additional CPD activities along with normal professional commitments for teachers [86]. The authors recommended developing strategies for improving leadership for such complex interventions. Other issues that hinder delivery through teachers in schools include a lack of time or training and uncertainty about their ability and role. Many teachers believed that obesity is a condition that requires treatment [87]. School teachers and personnel often receive little or no training in nutrition or obesity prevention techniques [20, 88, 89]. Providing CPD for teachers for intervention delivery can help them to feel more confident and is part of adopting a healthpromoting schools approach that encourages engaging parents and communities [20].

According to the health promoting schools framework of the International Union for Health Promotion and Education (IUHPE), schools have an essential role to play in health education for children and young people [90]. What is less clear is how best to engage with schools and provide evidence-based effective interventions. Our findings suggest that those interventions which showed improvement in BMI outcomes, worked with the school workforce to deliver the intervention. Hence, future interventions could benefit from planning with the education system, and from capitalizing on the expertise of teachers to best deliver messages and engage young people with interventions.

\section{Strengths and limitations}

Other systematic reviews have previously investigated the effectiveness of school-based obesity prevention in children and adolescents; however, this paper is the first to synthesize evidence for BMI outcomes, exclusively in adolescents. Previous reviews explored a broader age range (children and adolescents), different settings (global), or types of interventions (e.g. school and communities). While these reviews helped in providing an overview of the types and effectiveness of interventions for children and teenagers, our review provides in depth information on school-based interventions along with specific recommendations for school stakeholders. The review followed standardized guidance for conducting systematic reviews (full PRISMA checklist in supplementary material) and a rigorous assessment of risk of bias by two independent researchers and reported a detailed narrative synthesis of included studies. A random effects model was used for meta-analysis given the heterogeneous nature of the included studies. Only adjusted results were used for the meta-analysis to reduce bias. Due to the small number of studies eligible for the final meta-analysis, non-RCTs were also included to cover the evidence available. We constructed a funnel plot for the studies included within the meta-analysis. This suggested a degree of publication bias which might have led to over-estimation of effect size within the metaanalysis. The exclusion of studies that considered other anthropometric outcomes such as body fat percentage and waist circumference is an obvious limitation. Though these important anthropometric outcomes predict future risk of NCDs, systematic reviews have shown that the use of BMI to define obesity in children and adolescents is highly specific, albeit with low to moderate sensitivity [91]. To overcome this issue, in the metaanalysis we focused on BMI z-score over absolute BMI or change in BMI, which do not account for adolescent age [92]. Finally, as unpublished analyses, conference proceedings and grey literature were not reviewed, there is a possibility that other school-based interventions were overlooked. Finally, the generalisability of our findings may be limited to high income settings. Although these findings are more directly applicable to interventions developed in school-based settings, the insights will be of use to shape interventions aimed at adolescents.

\section{Recommendations and implications for research and public health}

A detailed analysis of the content of the interventions and comparison based on components was not feasible, as studies often did not include adequate details on these factors in their papers. Future publications of RCTs should consider using standardized ways of reporting intervention details and results, for example using the Template for Intervention Description and Replication (TIDieR) checklist and guide [93]. This improves completeness of reporting for individual study evaluations and further assists reviewers to collate and synthesize the findings. Similarly, future RCTs can consider including mediation analysis of behaviour change components in complex interventions.

Systematic reviews have often been criticized for inadequate consideration of the contexts in which interventions are delivered [94]. Contextual and cultural factors, education systems and prevalence of malnutrition can 
influence the delivery and uptake of interventions and their effectiveness. Hence, only high-income countries were included in this review. This could have led to omission of recent interventions in LMICs using multicomponent behaviour change theories and we recommend that research already conducted in LMICs needs to be reviewed, considering the co-existence of other forms of malnutrition and micronutrient deficiencies with childhood overweight and obesity, to identify the best platforms for interventions in such countries [95]. Similarly, in high-income settings, childhood obesity is often associated with lower SES and poor food environment [96]. While some studies in this review specifically targeted student/ schools from deprived areas [42, 47] future studies need to consider the barriers faced by students from schools in low-income areas who tend to have poorer diets and low physical activity levels use this information to develop targeted interventions. Policies for school health should consider including health education for obesity prevention in personal social health education curriculum, for example, to support the wider public health strategies for obesity prevention. While our review shows that shortterm outcomes for BMI can be modified through schoolbased interventions, further studies need to assess the long-term effects of these interventions and consider the sustainability and implementation at a population level.

\section{Conclusions}

The findings of this systematic review have implications for research and policy in high-income countries, to improve BMI outcomes in adolescence. Overall, our results suggest that school-based health education interventions could potentially help in improving BMI outcomes in the adolescent age group. Interventions should target the biological, psychosocial, environmental and behavioural influences on diet and physical activity. As many of the faceto-face interventions were effective, policy-makers could consider supporting schools to find ways to enable such interventions. Most school-based interventions were delivered by teachers and, including a CPD programme could improve teachers' confidence in delivering interventions. Alongside schools, parents should be engaged by adopting multi-component strategies to prevent obesity and overweight in adolescents. The research community should facilitate stronger working relationships with and between public health and education teams.

\section{Supplementary Information}

The online version contains supplementary material available at https://doi. org/10.1186/s12966-020-01065-9.

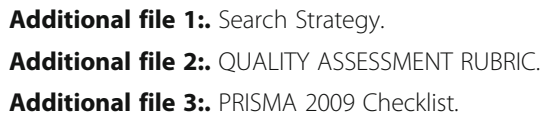

\section{Abbreviations}

BMI: Body Mass Index; CPD: Continuing Professional Development;

CRD: Centre for Reviews and Dissemination; HEIA: Health in Adolescents; LMIC: Low- and Middle-Income Countries; NCD: Non-Communicable Disease; PRISMA: Preferred Reporting Items for Systematic reviews and Meta-Analysis; RCT: Randomised Controlled Trial; SES: Socioeconomic Status

\section{Acknowledgements}

We also thank Liz Payne and Paula Sands for their assistance with the search strategy.

\section{Authors' contributions}

JB, KWT, MH, HMI, PHJ and CMJ conceptualized the paper. PHJ, CMJ, JB, KWT, MB planned the methodology. PHJ, CMJ, MB investigated and screened the articles and conducted data extraction and quality assessment. $\mathrm{HMI}$ and CP conducted the meta-analysis. PHJ and CMJ drafted the manuscript and all authors contributed to the review and revision of the final paper. All authors have approved the manuscript and consented for submission.

\section{Funding}

This paper presents independent research funded by the National Institute for Health Research (NIHR) under its Programme Grants for Applied Research Programme (Reference Number RP-PG-0216-20004). CMJ is funded by the EU Horizon 2020 programme LifeCycle under grant agreement No. 733206. PHJ and $\mathrm{MH}$ are funded in part by the British Heart Foundation (PG/14/33/30827) and Wessex Heartbeat. KWT is supported by the National Institute for Health Research, United Kingdom through the Southampton Biomedical Research Centre which also provides funds for work conducted by $\mathrm{MH}, \mathrm{HMI}$ and $\mathrm{CMJ}$. $\mathrm{HMI}$ and CMP are supported by the UK Medical Research Council and JB is partly funded by the UK Medical Research Council. The views expressed in this paper are those of the authors and not necessarily those of the funding organizations listed above.

\section{Availability of data and materials}

N/A

Ethics approval and consent to participate

N/A as systematic review of published literature.

\section{Consent for publication}

All authors consent to publication of the manuscript, figures, tables and supplementary material.

\section{Competing interests}

KWT, $\mathrm{MH}$ and $\mathrm{HI}$ are directors of LifeLab, a non-profit intervention at the University of Southampton and Southampton University Hospital, both registered charitable organizations. All other authors declare no conflicts of interest. The proposal for this review was registered with PROSPERO (ID: CRD42016053477)

\section{Author details}

${ }^{1}$ Academic Unit of Human Development and Health, Faculty of Medicine, University of Southampton, Southampton, UK. ${ }^{2} \mathrm{NIHR}$ Southampton Biomedical Research Centre, University Hospital Southampton NHS Foundation Trust, University of Southampton, Southampton, UK. ${ }^{3}$ Institute of Developmental Sciences, Faculty of Medicine, Southampton General Hospital, University of Southampton, Mail point 887, Tremona Road, Southampton SO16 6YD, UK. ${ }^{4}$ MRC Lifecourse Epidemiology Unit, University of Southampton, Southampton General Hospital, Level two, room 306, Tremona Road, Southampton SO16 6YD, UK. 5Southampton Education School, Faculty of Social Sciences, University of Southampton, Southampton, UK.

Received: 2 April 2020 Accepted: 30 November 2020

Published online: 04 January 2021

References

1. World Health Organisation. Factsheet on overweight and obesity2018 Aug 2018. Available from: http://www.who.int/news-room/fact-sheets/detail/ obesity-and-overweight. 
2. Lifshitz F, Rising R, Alemzadeh R. Obesity in children. Pediatr Endocrinol CRC Press. 2006:25-60.

3. Dietz WH. Health consequences of obesity in youth: childhood predictors of adult disease. Pediatrics. 1998;101(Supplement 2):518-25.

4. Wang Y, Lim H. The global childhood obesity epidemic and the association between socio-economic status and childhood obesity. Int Rev Psychiatry. 2012;24(3):176-88

5. Patton GC, Viner R. Pubertal transitions in health. Lancet Diabetes Endocrinol. 2007;369(9567):1130-9.

6. Reilly JJ, Methven E, McDowell ZC, Hacking B, Alexander D, Stewart L, et al. Health consequences of obesity. Arch Dis Child. 2003;88(9):748-52.

7. Sawyer SM, Afifi RA, Bearinger LH, Blakemore SJ, Dick B, Ezeh AC, et al. Adolescence: a foundation for future health. Lancet. 2012;379(9826):163040.

8. Alberga AS, Sigal RJ, Goldfield G, Prud'Homme D, Kenny GP. Overweight and obese teenagers: why is adolescence a critical period? Pediatr Obes. 2012;7(4):261-73.

9. Blakemore SJ, Mills KL. Is adolescence a sensitive period for sociocultural processing? Annu Rev Psychol. 2014;3(65):187-207.

10. Davis MM, Gance-Cleveland B, Hassink S, Johnson R, Paradis G, Resnicow K. Recommendations for prevention of childhood obesity. Pediatrics. 2007; 120(Supplement 4):S229-S53.

11. Pearson N, Biddle SJ. Sedentary behavior and dietary intake in children, adolescents, and adults: a systematic review. Am J Prev Med. 2011;41(2): 178-88.

12. Napier MA, Brown BB, Werner CM, Gallimore J. Walking to school: community design and child and parent barriers. J Environ Psychol. 2011; 31(1):45-51.

13. Story M, Neumark-Sztainer D, French S. Individual and environmental influences on adolescent eating behaviors. J Am Diet Assoc. 2002;102(3): S40-51.

14. Baranowski T, Cullen KW, Nicklas T, Thompson D, Baranowski J. Schoolbased obesity prevention: a blueprint for taming the epidemic. Am J Health Behav. 2002;26(6):486-93

15. Bleich $S N$, Vercammen KA, Zatz LY, Frelier JM, Ebbeling CB, Peeters A. Interventions to prevent global childhood overweight and obesity: a systematic review. Lancet Diabetes Endocrinol. 2017;6(4):332-46.

16. Kanekar A, Sharma M. Meta-analysis of school-based childhood obesity interventions in the UK and US. Int Q Community Health Educ. 2009;29(3): 241-56.

17. Kobes A, Kretschmer T, Timmerman G, Schreuder P. Interventions aimed at preventing and reducing overweight/obesity among children and adolescents: a meta-synthesis. Obes Rev. 2018;19(8):1065-79.

18. Lavelle HV, Mackay DF, Pell JP. Systematic review and meta-analysis of school-based interventions to reduce body mass index. J Public Health. 2012;34(3):360-9.

19. Lander N, Eather N, Morgan PJ, Salmon J, Barnett LM. Characteristics of teacher training in school-based physical education interventions to improve fundamental movement skills and/or physical activity: a systematic review. Sports Med. 2017;47(1):135-61.

20. Wang D. Stewart ds. The implementation and effectiveness of schoolbased nutrition promotion programmes using a health-promoting schools approach: a systematic review. Public Health Nutr. 2013;16(6): 1082-100.

21. Dobbins M, Husson H, DeCorby K, LaRocca RL. School-based physical activity programs for promoting physical activity and fitness in children and adolescents aged 6 to 18. Cochrane Database Syst Rev. 2013;2:CD007651.

22. Kriemler S, Meyer U, Martin E, van Sluijs EM, Andersen LB, Martin BW, et al. Effect of school-based interventions on physical activity and fitness in children and adolescents: a review of reviews and systematic update. $\mathrm{Br} J$ Sports Med. 2011:45(11):923-30.

23. Moores CJ, Bell LK, Miller J, Damarell RA, Matwiejczyk L, Miller MD. A systematic review of community-based interventions for the treatment of adolescents with overweight and obesity. Obes Rev. 2018;19(5):698-715.

24. UK Department of Health. Guidance. Childhood obesity: a plan for action 2017 Aug 2018. Available from: https:/www.gov.uk/government/ publications/childhood-obesity-a-plan-for-action/childhood-obesity-a-planfor-action.

25. Marks R. Health literacy: what is it and why should we care? In: Marks R, editor. Health literacy and school-based Education: Emerald Group Publishing; 2012
26. Nutbeam D, Harris E, Wise W. Theory in a nutshell: a practical guide to health promotion theories. McGraw-Hill. 2010.

27. Daniels SR, Arnett DK, Eckel RH, Gidding SS, Hayman LL, Kumanyika S, et al. Overweight in children and adolescents: pathophysiology, consequences, prevention, and treatment. Circulation. 2005;111(15):1999-2012.

28. Moher D, Liberati A, Tetzlaff J, Altman DG. Preferred reporting items for systematic reviews and meta-analyses: the PRISMA statement. Ann Intern Med. 2009;151(4):264-9.

29. Khan KS, Ter Riet G, Glanville J, Sowden AJ, Kleijnen J. Undertaking systematic reviews of research on effectiveness: CRD's guidance for carrying out or commissioning reviews NHS Centre for Reviews and Dissemination. 2001;No. 4 (2n).

30. World Health Organisation. Health topics: Adolescent Health Available 2018. Available from: http://www.who.int/topics/adolescent_health/en/.

31. Wang $Y$, Chen HJ. Use of percentiles and Z-scores in anthropometry. In: Handbook of anthropometry. New York, NY: Springer; 2012. p. 29-48.

32. The World Bank. World Bank Country and Lending Groups 2020 [August 2020]. Available from: https://datahelpdesk.worldbank.org/knowledgebase/ articles/906519-world-bank-country-and-lending-groups.

33. Green LW, Iverson DC. School health education. Annu Rev Public Health. 1982;3(1):321-38.

34. Amaro S, Viggiano A, Di Costanzo A, Madeo I, Viggiano A, Baccari ME, et al. Kaledo, a new educational board-game, gives nutritional rudiments and encourages healthy eating in children: a pilot cluster randomized trial. Eur J Pediatr. 2006;165(9):630-5.

35. Bonsergent E, Agrinier N, Thilly N, Tessier S, Legrand K, Lecomte E, et al. Overweight and obesity prevention for adolescents: a cluster randomized controlled trial in a school setting. Am J Prev Med. 2013;44(1):30-9.

36. Dewar DL, Morgan PJ, Plotnikoff RC, Okely AD, Collins CE, Batterham M, et al. The nutrition and enjoyable activity for teen girls study: a cluster randomized controlled trial. Am J Prev Med. 2013;45(3):313-7.

37. Ermetici F, Zelaschi RF, Briganti S, Dozio E, Gaeta M, Ambrogi F, et al. Association between a school-based intervention and adiposity outcomes in adolescents: the Italian "EAT" project. Obesity. 2016;24(3):687-95.

38. Fairclough SJ, Hackett AF, Davies IG, Gobbi R, Mackintosh KA, Warburton GL, et al. Promoting healthy weight in primary school children through physical activity and nutrition education: a pragmatic evaluation of the CHANGE! Randomised intervention study. BMC Public Health. 2013;13:626.

39. Foster GD, Sherman S, Borradaile KE, Grundy KM, Vander Veur SS, Nachmani J, et al. A policy-based school intervention to prevent overweight and obesity. Pediatrics. 2008;121(4):e794-802.

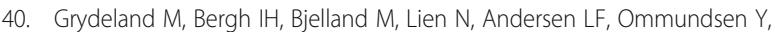
et al. Intervention effects on physical activity: the HEIA study - a cluster randomized controlled trial. Int J Behav Nutr Phys Act. 2013;10:17.

41. Lazorick S, Fang X, Hardison GT, Crawford Y. Improved body mass index measures following a middle school-based obesity intervention-the MATCH program. J Sch Health. 2015;85(10):680-7.

42. Lubans DR, Smith JJ, Plotnikoff RC, Dally KA, Okely AD, Salmon J, et al. Assessing the sustained impact of a school-based obesity prevention program for adolescent boys: the ATLAS cluster randomized controlled trial. Int J Behav Nutr Phys Act. 2016;13:92.

43. Millar L, Kremer P, de Silva-Sanigorski A, McCabe MP, Mavoa H, Moodie M, et al. Reduction in overweight and obesity from a 3-year community-based intervention in Australia: the 'It's your move!' project. Obes Rev. 2011; 12(Suppl 2):20-8.

44. Robbins LB, Pfeiffer KA, Maier KS, Lo YJ, Wesolek Ladrig SM. Pilot intervention to increase physical activity among sedentary urban middle school girls: a two-group pretest-posttest quasi-experimental design. J Sch Nurs. 2012;28(4):302-15.

45. Viggiano A, Viggiano E, Di Costanzo A, Viggiano A, Andreozzi E, Romano V, et al. Kaledo, a board game for nutrition education of children and adolescents at school: cluster randomized controlled trial of healthy lifestyle promotion. Eur J Pediatr. 2015;174(2):217-28.

46. Williamson DA, Champagne CM, Harsha DW, Han H, Martin CK, Newton RL $\mathrm{Jr}$, et al. Effect of an environmental school-based obesity prevention program on changes in body fat and body weight: a randomized trial. Obesity. 2012;20(8):1653-61.

47. Pfeiffer KA, Robbins LB, Ling J, Sharma DB, Dalimonte-Merckling DM, Voskuil $V R$, et al. Effects of the girls on the move randomized trial on adiposity and aerobic performance (secondary outcomes) in low-income adolescent girls. Pediatric Obesity. 2019;14(11):e12559. 
48. Bogart LM, Elliott MN, Cowgill BO, Klein DJ, Hawes-Dawson J, Uyeda K, et al. Two-year BMI outcomes from a school-based intervention for nutrition and exercise: a randomized trial. Pediatrics. 2016;137(5):e20152493.

49. Busch V, De Leeuw JR, Zuithoff NP, Van Yperen TA, Schrijvers AJ. A controlled health promoting school study in the Netherlands: effects after 1 and 2 years of intervention. Health Promot Pract. 2015;16(4):592-600.

50. Ezendam NP, Brug J, Oenema A. Evaluation of the web-based computertailored FATaintPHAT intervention to promote energy balance among adolescents: results from a school cluster randomized trial. Arch Pediatr Adolesc Med. 2012;166(3):248-55.

51. Graham DJ, Schneider M, Cooper DM. Television viewing: moderator or mediator of an adolescent physical activity intervention? Am J Health Promot. 2008;23(2):88-91.

52. HEALTHY Study Group. A school-based intervention for diabetes risk reduction. N Engl J Med. 2010;363(5):443-53.

53. Melnyk BM, Jacobson D, Kelly SA, Belyea MJ, Shaibi GQ, Small L, et al. Twelve-month effects of the COPE healthy lifestyles TEEN program on overweight and depressive symptoms in high school adolescents. J Sch Health. 2015;85(12):861-70.

54. Mihas C, Mariolis A, Manios Y, Naska A, Arapaki A, Mariolis-Sapsakos T, et al. Evaluation of a nutrition intervention in adolescents of an urban area in Greece: short- and long-term effects of the WYRONAS study. Public Health Nutr. 2010;13(5):712-9.

55. Neumark-Sztainer DR, Friend SE, Flattum CF, Hannan PJ, Story MT, Bauer KW, Feldman SB, Petrich CA. New moves-preventing weight-related problems in adolescent girls: a group-randomized study. Am J Prev Med. 2010;39(5): 421-32.

56. Peralta LR, Jones RA, Okely AD. Promoting healthy lifestyles among adolescent boys: the fitness improvement and lifestyle awareness program RCT. Prev Med. 2009;48(6):537-42.

57. Prins RG, Brug J, van Empelen P, Oenema A. Effectiveness of YouRAction, an intervention to promote adolescent physical activity using personal and environmental feedback: a cluster RCT. PLoS ONE [Electronic Resource]. 2012;7(3):e32682.

58. Rosenbaum M, Nonas C, Weil R, Horlick M, Fennoy I, Vargas I, et al. Schoolbased intervention acutely improves insulin sensitivity and decreases inflammatory markers and body fatness in junior high school students. J Clin Endocrinol Metab. 2006;92(2):504-8.

59. Singh AS, Chin APMJ, Brug J, van Mechelen W. Short-term effects of schoolbased weight gain prevention among adolescents. Arch Pediatr Adolesc Med. 2007;161(6):565-71

60. Wadolowska L, Hamulka J, Kowalkowska J, Ulewicz N, Hoffmann M, Gornicka $M$, et al. Changes in sedentary and active lifestyle, diet quality and body composition nine months after an education program in polish students aged 11-12 years: report from the ABC of healthy eating study. Nutrients. 2019;11(2):331.

61. Webber LS, Catellier DJ, Lytle LA, Murray DM, Pratt CA, Young DR, et al. Promoting physical activity in middle school girls: trial of activity for adolescent girls. Am J Prev Med. 2008;34(3):173-84

62. Wilksch SM, Paxton SJ, Byrne SM, Austin SB, McLean SA, Thompson KM, et al. Prevention across the Spectrum: a randomized controlled trial of three programs to reduce risk factors for both eating disorders and obesity. Psychol Med. 2015;45(9):1811-23.

63. Young DR, Phillips JA, Yu T, Haythornthwaite JA. Effects of a life skills intervention for increasing physical activity in adolescent girls. Arch Pediatr Adolesc Med. 2006;160(12):1255-61.

64. Fröberg A, Jonsson L, Berg C, Lindgren EC, Korp P, Lindwall M, et al. Effects of an empowerment-based health-promotion school intervention on physical activity and sedentary time among adolescents in a multicultural area. Int J Environ Res Public Health. 2018;15(11):2542.

65. Benitez-Andrades JA, Arias N, Garcia-Ordas MT, Martinez-Martinez M, Garcia-Rodriguez I. Feasibility of social-network-based eHealth intervention on the improvement of healthy habits among children. Sensors. 2020;20(5):04.

66. Robbins LB, Ling J, Clevenger K, Voskuil VR, Wasilevich E, Kerver JM, et al. A school- and home-based intervention to improve Adolescents' physical activity and healthy eating: a pilot study. J Sch Nurs. 2020;36(2):121-34.

67. Robbins LB, Ling J, Wen F. Attending after-school physical activity Club 2 days a week attenuated an increase in percentage body fat and a decrease in fitness among adolescent girls at risk for obesity. Am J Health Promot. 2020;34(5):500-4.
68. Smith JJ, Morgan PJ, Plotnikoff RC, Dally KA, Salmon J, Okely AD, Finn TL, Lubans DR. Smart-phone obesity prevention trial for adolescent boys in low-income communities: the ATLAS RCT. Pediatrics. 2014;134(3):e723-31.

69. Schneider M, Dunton GF, Bassin S, Graham DJ, Eliakim A, Cooper DM. Impact of a school-based physical activity intervention on fitness and bone in adolescent females. J Phys Act Health. 2007;4(1):17-29.

70. Bogart LM, Cowgill BO, Elliott MN, Klein DJ, Hawes-Dawson J, Uyeda K, et al. A randomized controlled trial of students for nutrition and eXercise: a communitybased participatory research study. J Adolesc Health. 2014;55(3):415-22.

71. Sallis JF, McKenzie TL, Alcaraz JE, Kolody B, Hovell MF, Nader P. R. Project SPARK. Effects of physical education on adiposity in children. Ann N Y Acad Sci. 1993;699:127-36.

72. Doak CM, Visscher TL, Renders CM, Seidell JC. The prevention of overweight and obesity in children and adolescents: a review of interventions and programmes. Obes Rev. 2006;7(1):111-36.

73. Katz DL, O'Connell M, Njike VY, Yeh MC, Nawaz H. Strategies for the prevention and control of obesity in the school setting: systematic review and meta-analysis. Int J Obes. 2008;32(12):1780.

74. Gonzalez-Suarez C, Worley A, Grimmer-Somers K, Dones V. School-based interventions on childhood obesity: a meta-analysis. Am J Prev Med. 2009; 37(5):418-27.

75. Dietz WH, Gortmaker SL. Preventing obesity in children and adolescents. Annu Rev Public Health. 2001;22(1):337-53.

76. Rose T, Barker M, Jacob CM, Morrison L, Lawrence W, Strömmer S, et al. A systematic review of digital interventions for improving the diet and physical activity behaviors of adolescents. J Adolesc Health. 2017;61 ((6)):669-77.

77. Ajie WN, Chapman-Novakofski KM. Impact of computer-mediated, obesityrelated nutrition education interventions for adolescents: a systematic review. J Adolesc Health. 2014;54(6):631-45.

78. Carbone ET, Zoellner JM. Nutrition and health literacy: A systematic review to inform nutrition research and practice. J Acad Nutr Diet. 2012;112(2):254-65.

79. Tate DF. Application of innovative technologies in the prevention and treatment of overweight in children and adolescents. Handbook of childhood and adolescent obesity. Boston, MA.: Springer; 2008. p. 387-404.

80. Woods-Townsend K, Leat H, Bay J, Bagust L, Davey H, Lovelock D, et al. LifeLab Southampton: a programme to engage adolescents with $\mathrm{DOHaD}$ concepts as a tool for increasing health literacy in teenagers-a pilot clusterrandomized control trial. J Dev Orig Health Dis. 2018;9(5):475-80.

81. Lazzer S, Boirie Y, Bitar A, Montaurier C, Vernet J, Meyer M, et al. Assessment of energy expenditure associated with physical activities in free-living obese and nonobese adolescents. Am J Clin Nutr. 2003;78(3):471-9.

82. Neumark-Sztainer D. Can we simultaneously work toward the prevention of obesity and eating disorders in children and adolescents? Int J Eat Disord. 2005;38(3):220-7.

83. Davies EL. "The monster of the month": teachers' views about alcohol within personal, social, health, and economic education (PSHE) in schools. Drugs and Alcohol Today. 2016;16(4):279-88.

84. Milliken-Tull A, McDonnell R. Alcohol and drug education in schools 2017. Available from: http://mentor-adepis.org/wp-content/uploads/2017/12/ Mentor-ADEPIS-Mapping-Report-October-2017.pdf.

85. Morgan PJ, Young MD, Smith JJ, Lubans DR. Targeted health behavior interventions promoting physical activity: a conceptual model. Exerc Sport Sci Rev. 2016:44(2):71-80.

86. Mathews LB, Moodie MM, Simmons AM, Swinburn BA. The process evaluation of It's your move!, an Australian adolescent community-based obesity prevention project. BMC Public Health. 2010;10(1):448.

87. Yager A, O'Dea JA. The role of teachers and other educators in the prevention of eating disorders and child obesity: what are the issues? Eat Disord. 2005:13:261-78.

88. Neumark-Sztainer D, Story M, Coller T. Perceptions of secondary school staff toward the implementation of school-based activities to prevent weightrelated disorders: a needs assessment. Am J Health Promot. 1999;13(3):153-6.

89. Stang JS, Story M, Kalina B. School-based weight management services: perceptions and practices of school nurses and administrators. Am J Health Promot. 1997;11(3):183-5.

90. International Union for Health Promotion and Education. Promoting Health in Schools: From evidence to Action 2010 [Available from: http://www. iuhpe.org/images/PUBLICATIONS/THEMATIC/HPS/Evidence-Action_ENG.pdf.

91. Reilly JJ, El-Hamdouchi A, Diouf A, Monyeki A, Somda SA. Determining the worldwide prevalence of obesity. Lancet. 2018:391(10132):1773-4. 
92. Nooyens AC, Koppes LL, Visscher TL, Twisk JW, Kemper HC, Schuit AJ, et al. Adolescent skinfold thickness is a better predictor of high body fatness in adults than is body mass index: the Amsterdam Growth and Health Longitudinal Study. Am J Clin Nutr. 2007:85(6):1533-9.

93. Hoffmann TC, Glasziou PP, Boutron I, Milne R, Perera R, Moher D, et al. Better reporting of interventions: template for intervention description and replication (TIDieR) checklist and guide. BMJ. 2014;348:g1687.

94. Rycroft-Malone J, McCormack B, Hutchinson AM, DeCorby K, Bucknall TK, Kent B, et al. Realist synthesis: illustrating the method for implementation research. Implement Sci. 2012;7(1):33.

95. Klingberg S, Draper CE, Micklesfield LK, Benjamin-Neelon SE, van Sluijs EM. Childhood obesity prevention in Africa: a systematic review of intervention effectiveness and implementation. Int J Environ Res Public Health. 2019; 16(7):1212.

96. Bann D, Johnson W, Li L, Kuh D, Hardy R. Socioeconomic inequalities in childhood and adolescent body-mass index, weight, and height from 1953 to 2015: an analysis of four longitudinal, observational, British birth cohort studies. Lancet Public Health. 2018;3(4):e194-203.

\section{Publisher's Note}

Springer Nature remains neutral with regard to jurisdictional claims in published maps and institutional affiliations.

Ready to submit your research? Choose BMC and benefit from:

- fast, convenient online submission

- thorough peer review by experienced researchers in your field

- rapid publication on acceptance

- support for research data, including large and complex data types

- gold Open Access which fosters wider collaboration and increased citations

- maximum visibility for your research: over $100 \mathrm{M}$ website views per year

At BMC, research is always in progress.

Learn more biomedcentral.com/submissions 